\title{
MEAN CURVATURE FLOW IN HIGHER CODIMENSION - INTRODUCTION AND SURVEY -
}

\author{
KNUT SMOCZYK
}

\begin{abstract}
In this text we outline the major techniques, concepts and results in mean curvature flow with a focus on higher codimension. In addition we include a few novel results and some material that cannot be found elsewhere.
\end{abstract}

\section{Mean Curvature Flow}

Mean curvature flow is perhaps the most important geometric evolution equation of submanifolds in Riemannian manifolds. Intuitively, a family of smooth submanifolds evolves under mean curvature flow, if the velocity at each point of the submanifold is given by the mean curvature vector at that point. For example, round spheres in euclidean space evolve under mean curvature flow while concentrically shrinking inward until they collapse in finite time to a single point, the common center of the spheres.

Mullins [Mul56] proposed mean curvature flow to model the formation of grain boundaries in annealing metals. Later the evolution of submanifolds by their mean curvature has been studied by Brakke Bra78] from the viewpoint of geometric measure theory. Among the first authors who studied the corresponding nonparametric problem were Temam [Tem76] in the late 1970's and Gerhardt Ger80] and Ecker Eck82 in the early 1980's. Pioneering work was done by Gage Gag84, Gage \& Hamilton GH86 and Grayson Gra87 who proved that the curve shortening flow (more precisely, the "mean" curvature flow of curves in $\mathbb{R}^{2}$ ) shrinks embedded closed curves to "round" points. In his seminal paper Huisken Hui84 proved that closed convex hypersurfaces in euclidean space $\mathbb{R}^{m+1}, m>1$ contract to single round points in finite time (later he extended his result to hypersurfaces in Riemannian manifolds that satisfy a suitable stronger convexity, see Hui86). Then, until the mid 1990's, most authors who studied mean curvature flow mainly considered hypersurfaces, both in euclidean and Riemannian manifolds, whereas mean curvature flow in higher codimension did not play a great role. There are various reasons for this, one of them is certainly the much different geometric situation of submanifolds in higher codimension since the normal bundle and the second fundamental tensor

Date: May 3, 2011.

2000 Mathematics Subject Classification. Primary 53C44;

Key words and phrases. introduction, survey, mean curvature flow, codimension, Lagrangian.

This survey is a contribution within the framework of the priority program "Globale Differentialgeometrie", DFG-SPP 1154, supported by the German Science Foundation (DFG). 
are more complicated. But also the analysis becomes more involved and the algebra of the second fundamental tensor is much more subtle since for hypersurfaces there usually exist more scalar quantities related to the second fundamental form than in case of submanifolds in higher codimension. Some of the results previously obtained for mean curvature flow of hypersurfaces carry over without change to submanifolds of higher codimension but many do not and in addition even new phenomena occur.

Among the first results in this direction are the results on mean curvature flow of space curves by Altschuler and Grayson [Alt91, AG92, measure-theoretic approaches to higher codimension mean curvature flows by Ambrosio \& Soner AS97, existence and convergence results for the Lagrangian mean curvature flow Smo96, Smo00, Smo02, TY02, mean curvature flow of symplectic surfaces in codimension two CL04,Wan02 and long-time existence and convergence results of graphic mean curvature flows in higher codimension CLT02, SW02, Smo04, Wan02, Xin08. Recently there has been done quite some work on the formation and classification of singularities in mean curvature flow Anc06, CL10, CCH09a, CSS07,CM09, GSSZ07, HL09, HS09, JLT10, LS10a, LS10b, LXYZ11, SW03, partially motivated by Hamilton's and Perelman's [Ham95a, Per02, Per03a, Per03b] work on the Ricci flow that in many ways behaves akin to the mean curvature flow and vice versa.

The results in mean curvature flow can be roughly grouped into two categories: The first category contains results that hold (more or less) in general, i.e. that are independent of dimension, codimension or the ambient space. In the second class we find results that are adapted to more specific geometric situations, like results for hypersurfaces, Lagrangian or symplectic submanifolds, graphs, etc..

Our aim in this article is twofold. We first want to summarize the most important properties of mean curvature flow that hold in any dimension, codimension and ambient space (first category). In the second part of this exposition we will give a - certainly incomplete and not exhaustive -, overview on more specific results in higher codimension, like an overview on the Lagrangian mean curvature flow or the mean curvature flow of graphs (part of the second category). Graphs and Lagrangian submanifolds certainly form the best understood subclasses of mean curvature flow in higher codimension.

In addition this article is intended as an introduction to mean curvature flow for the beginner and we will derive the most relevant geometric structure and evolution equations in a very general but consistent form that is rather hard to find in the literature. However, there are several nice monographs on mean curvature flow, a well written introduction to the regularity of mean curvature flow of hypersurfaces is Eck04. For the curve shortening flow see [CZ01. For mean curvature flow in higher codimension there exist some lecture notes by Wang Wan08b].

Let us now turn our attention to the mathematical definition of mean curvature flow. Suppose $M$ is a differentiable manifold of dimension $m, T>0$ a real number and $F: M \times[0, T) \rightarrow(N, g)$ a smooth time dependent family of immersions of $M$ into a Riemannian manifold $(N, g)$ of dimension $n$, i.e. $F$ is smooth and each

$$
F_{t}: M \rightarrow N, \quad F_{t}(p):=F(p, t), \quad t \in[0, T)
$$


is an immersion. If $F$ satisfies the evolution equation

$$
\frac{d F}{d t}(p, t)=\vec{H}(p, t), \quad \forall p \in M, t \in[0, T),
$$

where $\vec{H}(p, t) \in T_{F(p, t)} N$ is the mean curvature vector of the immersion $F_{t}$ at $p$ (or likewise of the submanifold $U_{t}:=F_{t}(U)$ at $F_{t}(p)$, if for some $U \subset M, F_{t \mid U}$ is an embedding), then we say that $M$ evolves by mean curvature flow in $N$ with initial data $F_{0}: M \rightarrow N$. As explained in section 2.1, the mean curvature vector field can be defined for any immersion into a Riemannian manifold (or more generally for any space-like immersion into a pseudo-Riemannian manifold; in this survey we will restrict to the Riemannian mean curvature flow) and it is the negative $L^{2}$-gradient of the volume functional vol $: \mathscr{I} \rightarrow \mathbb{R}$ on the space $\mathscr{I}$ of immersions of $M$ into $(N, g)$. Hence mean curvature flow is the steepest descent or negative $L^{2}$-gradient flow of the volume functional and formally equation (MCF) makes sense for any immersed submanifold in a Riemannian manifold. Therefore, following Hadamard, given an initial immersion $F_{0}: M \rightarrow N$ one is interested in the well-posedness of equation (MCF in the sense of

I.) Does a solution exist?

II.) Is it unique?

III.) Does it behave continuously in some suitable topology?

In addition, once short-time existence is established on some maximal time interval $[0, T), T \in(0, \infty]$, one wants to study the behavior of the flow and in particular of the evolving immersed submanifolds $M_{t}:=F_{t}(M)$ as $t \rightarrow T$. Either singularities of some kind will form and one might then study the formation of singularities in more details - with possible significant geometric implications - or the flow has a long-time solution. In such a case convergence to some nice limit (e.g. stationary, i.e. a limit with vanishing mean curvature) would be rather expected but in general will not hold a-priori.

In the most simplest case, i.e. if the dimension of $M$ is one, mean curvature flow is called curve shortening flow. In many contributions to the theory of mean curvature flow one assumes that $M$ is a smooth closed manifold. The reason is, that one key technique in mean curvature flow (or more generally in the theory of parabolic geometric evolution equations) is the application of the maximum principle and in absence of compactness the principle of "first time violation" of a stated inequality simply does not hold. But even for complete non-compact submanifolds there are powerful techniques, similar to the maximum principle, that can be applied in some situations. In the complete case one of the most important tools is the monotonicity formula found by Huisken [Hui90, Ecker \& Huisken [EH89] and Hamilton [Ham93] and that equally well applies to mean curvature flow in higher codimension. Ecker Eck01 proved a beautiful local version of the monotonicity formula for hypersurfaces and another local monotonicity for evolving Riemannian manifolds has been found recently by Ecker, Knopf, Ni and Topping EKNT08.

There are some very important contributions to the regularity theory of mean curvature flow by White Whi05, Whi09 that apply in all codimensions. For example in Whi05 he proves uniform curvature bounds of the euclidean mean curvature flow in regions of space-time where the Gaussian density ratios are close to 1 . With this 
result one can often exclude finite time singularities and prove long-time existence of the flow (see for example Wan02, MW09]).

For simplicity and since some techniques and results do not hold for complete noncompact manifolds we will always assume in this article, unless otherwise agreed, that $M$ is an oriented closed smooth manifold.

The organization of the survey is as follows: In section 2 we will review the geometric structure equations for immersions in Riemannian manifolds and we will introduce most of our terminology and notations that will be used throughout the paper. In particular we will mention the explicit formulas in the case of Lagrangian submanifolds in Kähler-Einstein manifolds. For most computations we will use the Ricci calculus and apply the Einstein convention to sum over repeated indices. In section 3 we will summarize those results that hold in general (first category). The section is subdivided into four subsections. In the first subsection 3.1 we will show that the mean curvature flow is a quasilinear (degenerate) parabolic system and we will treat the existence and uniqueness problem. In subsection 3.2 we derive the evolution equations of the most important geometric quantities in the general situation, i.e. for immersions in arbitrary Riemannian manifolds. In this general form these formulas are hard to find in the literature and one can later easily derive all related evolution equations from them that occur in special situations like evolution equations for tensors that usually appear in mean curvature flow of hypersurfaces, Lagrangian submanifolds or graphs. In subsection 3.3 we recall general results concerning long-time existence of solutions. In the final subsection 3.4 of this section we explain the two types of singularities that appear in mean curvature flow and discuss some rescaling techniques. Moreover we will recall some of the results that have been obtained in the classification of solitons. Section 4 is on more specific results in higher codimension, the first subsection treats the Lagrangian mean curvature flow and in the last and final subsection of this article we give an overview of the results in mean curvature flow of graphs.

\section{The GeOMETRY of IMMERSions}

\subsection{Second fundamental form and mean curvature vector.}

In this subsection we recall the definition of the second fundamental form and mean curvature vector of an immersion and we will introduce most of our notation.

Let $F: M \rightarrow(N, g)$ be an immersion of an $m$-dimensional differentiable manifold $M$ into a Riemannian manifold $(N, g)$ of dimension $n$, i.e. $F$ is smooth and the pull-back $F^{*} g$ defines a Riemannian metric on $M$. The number $k:=n-m \geq 0$ is called the codimension of the immersion.

For $p \in M$ let

$$
T_{p}^{\perp} M:=\left\{\nu \in T_{F(p)} N: g\left(\nu, D F_{\mid p}(W)\right)=0, \forall W \in T_{p} M\right\}
$$

denote the normal space of $M$ at $p$ and $T^{\perp} M$ the associated normal bundle. By definition, the normal bundle of $M$ is a sub-bundle of rank $k$ of the pull-back bundle 
$F^{*} T N=\bigcup_{p \in M} T_{F(p)} N$ over $M$. Using the differential of $F$ we thus have a splitting

$$
T_{F(p)} N=D F_{\mid p}\left(T_{p} M\right) \oplus T_{p}^{\perp} M .
$$

The differential $D F$ can be considered as a 1-form on $M$ with values in $F^{*} T N$, i.e.

$$
\begin{array}{r}
D F \in \Gamma\left(F^{*} T N \otimes T^{*} M\right)=: \Omega^{1}\left(M, F^{*} T N\right), \\
T_{p} M \ni V \mapsto D F_{\mid p}(V) \in T_{F(p)} N .
\end{array}
$$

The Riemannian metric $F^{*} g$ is also called the first fundamental form on $M$. In an obvious way the metrics $g$ and $F^{*} g$ induce Riemannian metrics on all bundles formed from products of $T M, T^{*} M, T^{\perp} M, F^{*} T N, T N$, and $T^{*} N$ and in the sequel we will often denote all such metrics simply by the usual brackets $\langle\cdot, \cdot\rangle$ for an inner product.

Similarly the Levi-Civita connection $\nabla$ on $(N, g)$ induces connections on the bundles $T M, T^{*} M, T^{\perp} M, F^{*} T N$ and products hereof. Since the precise definition of these connections will be crucial in the understanding of the second fundamental form, the mean curvature vector and later also of the evolution equations, we will briefly recall them. The connection $\nabla^{T M}$ on $T M$ can be obtained in two equivalent ways: either as the Levi-Civita connection of the induced metric $F^{*} g$ on $T M$ or else by projection of the ambient connection to the tangent bundle, more precisely via the formula

$$
D F\left(\nabla_{X}^{T M} Y\right):=\nabla_{D F(X)}^{T} \overline{D F(Y)}, \quad X, Y \in T M,
$$

where ${ }^{\top}$ denotes the projection onto $D F(T M)$ and $\overline{D F(Y)}$ is an arbitrary (local) smooth extension of $D F(Y)$. The connection $\nabla^{T^{*} M}$ on $T^{*} M$ is then simply given by the dual connection of $\nabla^{T M}$. Similarly one obtains the connection $\nabla^{F^{*} T N}$ on $F^{*} T N$ via the formula

$$
\nabla_{X}^{F^{*} T N} V:=\nabla_{D F(X)} \bar{V}
$$

for any smooth section $V \in \Gamma\left(F^{*} T N\right)$ and finally the connection $\nabla^{\perp}$ on the normal bundle is given by projection

$$
\nabla_{X}^{\perp} \nu:=\left(\nabla_{X}^{F^{*} T N} \nu\right)^{\perp}
$$

for $\nu \in \Gamma\left(T^{\perp} M\right) \subset \Gamma\left(F^{*} T N\right)$. Since the connections $\nabla^{T M}, \nabla^{T^{*} M}, \nabla^{F^{*} T N}$ and their associated product connections on product bundles over $M$ formed from the factors $T M, T^{*} M, F^{*} T N$ are induced by $\nabla$, it is common (and sometimes confusing) to denote all of them by the same symbol $\nabla$. Since $T^{\perp} M$ is a sub-bundle of $F^{*} T N$, one can consider a section $\nu \in \Gamma\left(T^{\perp} M\right)$ also as an element of $\Gamma\left(F^{*} T N\right)$ and hence one can apply both connections $\nabla^{\perp}$ and $\nabla=\nabla^{F^{*} T N}$ to them, i.e. we will write $\nabla_{X} \nu\left(=\nabla_{X}^{F^{*} T N} \nu\right)$, if we consider $\nu$ as a section in $F^{*} T N$ and $\nabla_{X}^{\frac{1}{X}} \nu$, if $\nu$ is considered as a section in the normal bundle $T^{\perp} M$. The same holds, if we consider sections in product bundles that contain $T^{\perp} M$ as a factor.

If we apply the resulting connection $\nabla$ on $F^{*} T N \otimes T^{*} M$ to $D F$, we obtain - by definition - the second fundamental tensor

$$
A:=\nabla D F \in \Gamma\left(F^{*} T N \otimes T^{*} M \otimes T^{*} M\right) .
$$

It is then well-known that the second fundamental tensor is symmetric

$$
A(X, Y)=\left(\nabla_{X} D F\right)(Y)=\left(\nabla_{Y} D F\right)(X)=A(Y, X)
$$


and normal in the sense that

$$
\langle A(X, Y), D F(Z)\rangle=0, \quad \forall X, Y, Z \in T M .
$$

Therefore in particular $A \in \Gamma\left(T^{\perp} M \otimes T^{*} M \otimes T^{*} M\right)$.

Taking the trace of $A$ gives the mean curvature vector field

$$
\vec{H}:=\operatorname{trace} A=\sum_{i=1}^{m} A\left(e_{i}, e_{i}\right),
$$

where $\left(e_{i}\right)_{i=1, \ldots, m}$ is an arbitrary orthonormal frame of $T M$. Hence, since $A$ is normal, we obtain a canonical section $\vec{H} \in \Gamma\left(T^{\perp} M\right)$ in the normal bundle of the immersion $F: M \rightarrow N$.

\subsection{Structure equations.}

The second fundamental tensor is a curvature quantity that determines how curved the immersed submanifold $F(M)$ given by an immersion $F: M \rightarrow N$ lies within the ambient manifold $(N, g)$. According to this we have a number of geometric equations that relate the second fundamental tensor to the intrinsic curvatures of $\left(M, F^{*} g\right)$ and $(N, g)$.

Let $\nabla$ be a connection on a vector bundle $E$ over a smooth manifold $M$. Our convention for the curvature tensor $R^{E, \nabla} \in \Omega^{2}(M, E)$ w.r.t. $\nabla$ is

$$
R^{E, \nabla}(X, Y) \sigma:=\left(\nabla_{X} \nabla_{Y}-\nabla_{Y} \nabla_{X}-\nabla_{[X, Y]}\right) \sigma, \quad \forall X, Y \in T M, \sigma \in \Gamma(E) .
$$

Moreover, if $E$ is a bundle with bundle metric $\langle\cdot, \cdot\rangle$, then we set

$$
R^{E, \nabla}(\mu, \sigma, X, Y):=\left\langle\mu, R^{E, \nabla}(X, Y) \sigma\right\rangle, \quad \forall X, Y \in T M, \sigma, \mu \in E .
$$

We denote the curvature tensors $R^{T M, \nabla}$ and $R^{T N, \nabla}$ by $R^{M}$ resp. $R^{N}$. Letting

$$
\left(\nabla_{X} A\right)(Y, V):=\nabla_{X}(A(Y, V))-A\left(\nabla_{X} Y, V\right)-A\left(Y, \nabla_{X} V\right)
$$

the Codazzi equation is

$$
\begin{aligned}
& \left(\nabla_{X} A\right)(Y, V)-\left(\nabla_{Y} A\right)(X, V) \\
& =R^{N}(D F(X), D F(Y)) D F(V)-D F\left(R^{M}(X, Y) V\right) .
\end{aligned}
$$

Note that $\nabla$ denotes the full connection, i.e. here we consider $A$ as a section in $F^{*} T N \otimes T^{*} M \otimes T^{*} M$ and not in $T^{\perp} M \otimes T^{*} M \otimes T^{*} M$. Later we will sometimes consider $A$ as a section in $T^{\perp} M \otimes T^{*} M \otimes T^{*} M$ and then we will also use the connection on the normal bundle instead, so that in this case we write $\left(\nabla_{X}^{\perp} A\right)(Y, V)=\left(\left(\nabla_{X} A\right)(Y, V)\right)^{\perp}$. In terms of $\nabla^{\perp}$ the Codazzi equation becomes

$$
\left(\nabla_{X}^{\perp} A\right)(Y, V)-\left(\nabla_{Y}^{\perp} A\right)(X, V)=\left(R^{N}(D F(X), D F(Y)) D F(V)\right)^{\perp} .
$$

From

$$
\langle A(Y, V), D F(W)\rangle=0, \quad \forall Y, V, W \in T M
$$

we get

$$
\left\langle\left(\nabla_{X} A\right)(Y, V), D F(W)\right\rangle=-\langle A(Y, V), A(X, W)\rangle .
$$


From these equations we obtain Gauß equation (Theorema Egregium):

$$
\begin{aligned}
R^{M}(X, Y, V, W) & =R^{N}(D F(X), D F(Y), D F(V), D F(W)) \\
& +\langle A(X, V), A(Y, W)\rangle-\langle A(X, W), A(Y, V)\rangle .
\end{aligned}
$$

Finally, we have Ricci's equation. If $\nu \in T^{\perp} M$ and $X, Y \in T M$ then the following holds:

$$
\begin{aligned}
& R^{\perp}(X, Y) \nu=\left(R^{N}(D F(X), D F(Y)) \nu\right)^{\perp} \\
& -\sum_{i=1}^{m}\left(\left\langle\nu, A\left(X, e_{i}\right)\right\rangle A\left(Y, e_{i}\right)-\left\langle\nu, A\left(Y, e_{i}\right)\right\rangle A\left(X, e_{i}\right)\right),
\end{aligned}
$$

where $\left(e_{i}\right)_{i=1, \ldots, m}$ is an arbitrary orthonormal frame of $T M$ and $R^{\perp}=R^{T^{\perp} M, \nabla^{\perp}}$ denotes the curvature tensor of the normal bundle of $M$. Note that the Codazzi equation is useless in dimension one (i.e. for curves) and that Ricci's equation is useless for hypersurfaces, i.e. in codimension one.

\subsection{Tensors in local coordinates.}

For computations one often needs local expressions of tensors. Whenever we use local expressions and $F: M \rightarrow N$ is an immersion we make the following general assumptions and notations

i) $(U, x, \Omega)$ and $(V, y, \Lambda)$ are local coordinate charts around $p \in U \subset M$ and $F(p) \in V \subset N$ such that $F_{\mid U}: U \rightarrow F(U)$ is an embedding and such that $F(U) \subset V$.

ii) From the coordinate functions

$$
\left(x^{i}\right)_{i=1, \ldots, m}: U \rightarrow \Omega \subset \mathbb{R}^{m}, \quad\left(y^{\alpha}\right)_{\alpha=1, \ldots, n}: V \rightarrow \Lambda \subset \mathbb{R}^{n}
$$

we obtain a local expression for $F$,

$$
y \circ F \circ x^{-1}: \Omega \rightarrow \Lambda, \quad F^{\alpha}:=y^{\alpha} \circ F \circ x^{-1}, \quad \alpha=1, \ldots, n .
$$

iii) The Christoffel symbols of the Levi-Civita connections on $M$ resp. $N$ will be denoted

$$
\Gamma_{j k}^{i}, \quad i, j, k=1, \ldots, m, \quad \text { resp. } \quad \Gamma_{\beta \gamma}^{\alpha}, \quad \alpha, \beta, \gamma=1, \ldots, n .
$$

iv) All indices referring to $M$ will be denoted by Latin minuscules and those related to $N$ by Greek minuscules. Moreover, we will always use the Einstein convention to sum over repeated indices from 1 to the respective dimension.

Then the local expressions for $g, D F, F^{*} g$ and $A$ are

$$
\begin{gathered}
g=g_{\alpha \beta} d y^{\alpha} \otimes d y^{\beta}, \\
D F=F_{i}^{\alpha} \frac{\partial}{\partial y^{\alpha}} \otimes d x^{i}, \quad F_{i}^{\alpha}:=\frac{\partial F^{\alpha}}{\partial x^{i}}, \\
F^{*} g=g_{i j} d x^{i} \otimes d x^{j}, \quad g_{i j}:=g_{\alpha \beta} F_{i}^{\alpha} F_{j}^{\beta},
\end{gathered}
$$

and

$$
A=A_{i j} d x^{i} \otimes d x^{j}=A^{\alpha}{ }_{i j} \frac{\partial}{\partial y^{\alpha}} \otimes d x^{i} \otimes d x^{j},
$$


where the coefficients $A^{\alpha}{ }_{i j}$ are given by Gauß' formula

$$
A^{\alpha}{ }_{i j}=\frac{\partial^{2} F^{\alpha}}{\partial x^{i} \partial x^{j}}-\Gamma_{i j}^{k} \frac{\partial F^{\alpha}}{\partial x^{k}}+\Gamma_{\beta \gamma}^{\alpha} \frac{\partial F^{\beta}}{\partial x^{i}} \frac{\partial F^{\gamma}}{\partial x^{j}} .
$$

Let $\left(g^{i j}\right)$ denote the inverse matrix of $\left(g_{i j}\right)$ so that $g^{i k} g_{k j}=\delta^{i}{ }_{j}$ gives the Kronecker symbol. $\left(g^{i j}\right)$ defines the metric on $T^{*} M$ dual to $F^{*} g$. For the mean curvature vector we get

$$
\vec{H}=H^{\alpha} \frac{\partial}{\partial y^{\alpha}}, \quad H^{\alpha}:=g^{i j} A^{\alpha}{ }_{i j} .
$$

Gauß' equation (8) now becomes

$$
R_{i j k l}=R_{\alpha \beta \gamma \delta} F_{i}^{\alpha} F^{\beta}{ }_{j} F^{\gamma}{ }_{k} F^{\delta}{ }_{l}+g_{\alpha \beta}\left(A^{\alpha}{ }_{i k} A^{\beta}{ }_{j l}-A^{\alpha}{ }_{i l} A^{\beta}{ }_{j k}\right),
$$

where the notation should be obvious, e.g.

$$
R_{i j k l}=R^{M}\left(\frac{\partial}{\partial x^{i}}, \frac{\partial}{\partial x^{j}}, \frac{\partial}{\partial x^{k}}, \frac{\partial}{\partial x^{l}}\right)
$$

and

$$
R_{\alpha \beta \gamma \delta}=R^{N}\left(\frac{\partial}{\partial y^{\alpha}}, \frac{\partial}{\partial y^{\beta}}, \frac{\partial}{\partial y^{\gamma}}, \frac{\partial}{\partial y^{\delta}}\right) .
$$

Note that the choice of the indices already indicates which curvature tensor is used. In addition we write

$$
\nabla A=\nabla_{i} A^{\alpha}{ }_{j k} \frac{\partial}{\partial y^{\alpha}} \otimes d x^{i} \otimes d x^{j} \otimes d x^{k},
$$

so that

$$
\left(\nabla_{\frac{\partial}{\partial x^{i}}} A\right)\left(\frac{\partial}{\partial x^{j}}, \frac{\partial}{\partial x^{k}}\right)=\nabla_{i} A^{\alpha}{ }_{j k} \frac{\partial}{\partial y^{\alpha}} .
$$

Similar notations will be used for other covariant derivatives, e.g. $\nabla_{i} \nabla_{j} T_{l}^{k}$ will denote the coefficients of the tensor $\nabla^{2} T$ with $T \in \Gamma\left(T M \otimes T^{*} M\right)=\operatorname{End}(T M)$. The Codazzi equation in local coordinates is

$$
\nabla_{i} A^{\alpha}{ }_{j k}-\nabla_{j} A^{\alpha}{ }_{i k}=R^{\alpha}{ }_{\beta \gamma \delta} F_{k}^{\beta} F_{i}^{\gamma} F_{j}^{\delta}-R_{k i j}^{l} F_{l}^{\alpha},
$$

where here and in the following all indices will be raised and lowered using the metric tensors, e.g.

$$
R_{\beta \gamma \delta}^{\alpha}=g^{\alpha \epsilon} R_{\epsilon \beta \gamma \delta}, \quad R_{k l}^{i j}=g^{i p} g^{j q} R_{k p l q} .
$$

Finally, if $\left(\nu_{A}\right)_{A=1, \ldots, k:=n-m}, \nu_{A}=\nu_{A}^{\alpha} \frac{\partial}{\partial y^{\alpha}}$, is a local trivialization of $T^{\perp} M$, then

$$
R^{\perp}\left(\frac{\partial}{\partial x^{i}}, \frac{\partial}{\partial x^{j}}\right) \nu_{A}=:\left(R^{\perp}\right)^{B}{ }_{A i j} \nu_{B}
$$

and Ricci's equation becomes

$$
\begin{aligned}
\left(R^{\perp}\right)^{B}{ }_{A i j} \nu_{B}^{\alpha}= & R^{\alpha}{ }_{\beta \gamma \delta} \nu_{A}^{\beta} F^{\gamma}{ }_{i} F_{j}^{\delta}-g^{k l} R_{\beta \gamma \delta}^{\epsilon} g_{\epsilon \sigma} \nu_{A}^{\beta} F^{\gamma}{ }_{i} F^{\delta}{ }_{j} F^{\sigma}{ }_{k} \\
& -g_{\beta \gamma} g^{k l}\left(\nu_{A}^{\beta} A_{i k}^{\gamma} A_{j l}^{\alpha}-\nu_{A}^{\beta} A_{j k}^{\gamma} A_{i l}^{\alpha}\right) .
\end{aligned}
$$


Using the rule for interchanging covariant derivatives and the structure equations one obtains Simons' identity

$$
\begin{aligned}
\nabla_{k} \nabla_{l} H^{\alpha}= & \Delta A^{\alpha}{ }_{k l}+\left(\nabla_{\epsilon} R^{\alpha}{ }_{\beta \gamma \delta}+\nabla_{\gamma} R^{\alpha}{ }_{\delta \beta \epsilon}\right) F_{i}^{\epsilon} F^{\beta}{ }_{l} F^{\gamma}{ }_{k} F^{\delta i} \\
& +R^{\alpha}{ }_{\beta \gamma \delta}\left(2 A^{\beta}{ }_{i k} F^{\gamma}{ }_{l} F^{\delta i}+2 A^{\beta}{ }_{i l} F^{\gamma}{ }_{k} F^{\delta i}\right. \\
& \left.+H^{\delta} F^{\beta}{ }_{l} F^{\gamma}{ }_{k}+A^{\gamma}{ }_{l k} F^{\beta}{ }_{i} F^{\delta i}\right) \\
& -\left(\nabla_{k} R^{p}{ }_{l}+\nabla_{l} R^{p}{ }_{k}-\nabla^{p} R_{k l}\right) F_{p}^{\alpha} \\
& +2 R_{k l}{ }^{i j}{ }^{\alpha}{ }^{\alpha}{ }_{i j}-R^{p}{ }_{k} A^{\alpha}{ }_{p l}-R^{p}{ }_{l} A^{\alpha}{ }_{p k},
\end{aligned}
$$

where $R_{i j}=g^{k l} R_{i k j l}$ denotes the Ricci curvature on $M$. If one multiplies Simons' identity (15) with $2 A_{\alpha}{ }^{k l}=2 g_{\alpha \epsilon} g^{k m} g^{l n} A_{m n}^{\epsilon}$, one gets

$$
\begin{aligned}
2\left\langle A, \nabla^{2} \vec{H}\right\rangle= & \Delta|A|^{2}-2|\nabla A|^{2} \\
& +2\left(\nabla_{\epsilon} R_{\alpha \beta \gamma \delta}+\nabla_{\gamma} R_{\alpha \delta \beta \epsilon}\right) F_{i}^{\epsilon} F^{\beta l} F^{\gamma k} F^{\delta i} A^{\alpha}{ }_{k l} \\
& +2 R_{\alpha \beta \gamma \delta} A^{\alpha k l}\left(4 A^{\beta}{ }_{i k} F^{\gamma}{ }_{l} F^{\delta i}+H^{\delta} F^{\beta}{ }_{l} F^{\gamma}{ }_{k}+A^{\gamma}{ }_{l k} F^{\beta}{ }_{i} F^{\delta i}\right) \\
& +4 R^{k i l j}\left\langle A_{i j}, A_{k l}\right\rangle-4 R^{i j}\left\langle A_{i k}, A_{j}{ }^{k}\right\rangle
\end{aligned}
$$

and then since

$$
\begin{aligned}
\nabla_{i} A_{k l} & =\nabla_{i}^{\perp} A_{k l}+g^{p q}\left\langle\nabla_{i} A_{k l}, F_{p}\right\rangle F_{q} \\
& =\nabla_{i}^{\perp} A_{k l}-g^{p q}\left\langle A_{k l}, \nabla_{i} F_{p}\right\rangle F_{q} \\
& =\nabla_{i}^{\perp} A_{k l}-g^{p q}\left\langle A_{k l}, A_{i p}\right\rangle F_{q}
\end{aligned}
$$

implies

$$
|\nabla A|^{2}=\left|\nabla^{\perp} A\right|^{2}+\left\langle A^{i j}, A^{k l}\right\rangle\left\langle A_{i j}, A_{k l}\right\rangle
$$

we obtain with Gauß' equation the second Simons' identity

$$
\begin{aligned}
2\left\langle A, \nabla^{2} \vec{H}\right\rangle= & \Delta|A|^{2}-2\left|\nabla^{\perp} A\right|^{2} \\
& +2\left\langle A^{i j}, A^{k l}\right\rangle\left\langle A_{i j}, A_{k l}\right\rangle-4\left\langle A^{k j}, A^{i l}\right\rangle\left\langle A_{i j}, A_{k l}\right\rangle \\
& -4\left\langle\vec{H}, A^{i j}\right\rangle\left\langle A_{i k}, A_{j}{ }^{k}\right\rangle+4\left\langle A^{i l}, A_{l}{ }^{j}\right\rangle\left\langle A_{i k}, A_{j}{ }^{k}\right\rangle \\
& +4 R_{\alpha \beta \gamma \delta} F^{\alpha}{ }_{k} F^{\beta}{ }_{i} F^{\gamma} F^{\delta}{ }_{j}\left(\left\langle A^{i j}, A^{k l}\right\rangle-g^{k l}\left\langle A^{i p}, A_{p}{ }^{j}\right\rangle\right) \\
& +2 R_{\alpha \beta \gamma \delta} A^{\alpha k l}\left(4 A^{\beta}{ }_{i k} F^{\gamma}{ }_{l} F^{\delta i}+F^{\beta}{ }_{l} F^{\gamma}{ }_{k} H^{\delta}+F^{\beta}{ }_{i} A^{\gamma}{ }_{l k} F^{\delta i}\right) \\
& +2\left(\nabla_{\epsilon} R_{\alpha \beta \gamma \delta}+\nabla_{\gamma} R_{\alpha \delta \beta \epsilon}\right) F^{\epsilon}{ }_{i} F^{\beta}{ }_{l} F^{\gamma}{ }_{k} F^{\delta i} A^{\alpha k l} .
\end{aligned}
$$

The second and third line can be further simplified, so that we get

$$
\begin{aligned}
2\left\langle A, \nabla^{2} \vec{H}\right\rangle= & \Delta|A|^{2}-2\left|\nabla^{\perp} A\right|^{2} \\
& +\left|\left\langle A_{i j}, A_{k l}\right\rangle-\left\langle A_{i l}, A_{j k}\right\rangle\right|^{2}+\left|A^{\alpha}{ }_{i k} A^{\beta}{ }_{j}{ }^{k}-A^{\beta}{ }_{i k} A^{\alpha}{ }_{j}{ }^{k}\right|^{2} \\
& +2\left|\left\langle\vec{H}, A_{i j}\right\rangle-\left\langle A_{i k}, A_{j}{ }^{k}\right\rangle\right|^{2}-2\left|\left\langle\vec{H}, A_{i j}\right\rangle\right|^{2} \\
& +4 R_{\alpha \beta \gamma \delta} F^{\alpha}{ }_{k} F^{\beta}{ }_{i} F^{\gamma}{ }_{l} F^{\delta}{ }_{j}\left(\left\langle A^{i j}, A^{k l}\right\rangle-g^{k l}\left\langle A^{i p}, A_{p}{ }^{j}\right\rangle\right) \\
& +2 R_{\alpha \beta \gamma \delta} A^{\alpha k l}\left(4 A^{\beta}{ }_{i k} F^{\gamma}{ }_{l} F^{\delta i}+F^{\beta}{ }_{l} F^{\gamma}{ }_{k} H^{\delta}+F^{\beta}{ }_{i} A^{\gamma}{ }_{l k} F^{\delta i}\right) \\
& +2\left(\nabla_{\epsilon} R_{\alpha \beta \gamma \delta}+\nabla_{\gamma} R_{\alpha \delta \beta \epsilon}\right) F^{\epsilon}{ }_{i} F^{\beta}{ }_{l} F^{\gamma}{ }_{k}{ }^{\delta i} A^{\alpha k l} .
\end{aligned}
$$


This last equation is useful to substitute terms in the evolution equation of $|A|^{2}$ (see subsection 3.2 below).

\subsection{Special situations.}

\subsubsection{Hypersurfaces.}

If $F: M \rightarrow N$ is an immersion of a hypersurface, then $n=m+1$ and one can define a number of scalar curvature quantities related to the second fundamental tensor of $M$. For simplicity assume that both $M$ and $N$ are orientable (otherwise the following computations are only local). Then there exists a unique normal vector field $\nu \in \Gamma\left(T^{\perp} M\right)$ - called the principle normal - such that for all $p \in M$ :

i) $\left|\nu_{\mid p}\right|=1, \nu_{\mid p} \in T_{p}^{\perp} M$

ii) If $e_{1}, \ldots, e_{m}$ is a positively oriented basis of $T_{p} M$, then

$$
D F\left(e_{1}\right), \ldots, D F\left(e_{m}\right), \nu_{\mid p}
$$

forms a positively oriented basis of $T_{F(p)} N$.

Using the principle normal $\nu$, one defines the (scalar) second fundamental form $h \in \Gamma\left(T^{*} M \otimes T^{*} M\right)$ by

$$
h(X, Y):=\langle A(X, Y), \nu\rangle
$$

and the scalar mean curvature $H$ by

$$
H:=\operatorname{trace} h
$$

so that

$$
A=\nu \otimes h, \quad \vec{H}=H \nu .
$$

The map

$$
\text { b }: T M \rightarrow T^{*} M, \quad V \mapsto V_{b}:=\langle V, \cdot\rangle
$$

is a bundle isomorphism with inverse denoted by

$$
\sharp: T^{*} M \rightarrow T M \text {. }
$$

This musical isomorphism can be used to define the Weingarten map

$$
\mathscr{W} \in \operatorname{End}(T M), \quad \mathscr{W}(X):=(h(X, \cdot))^{\sharp} .
$$

Since $h$ is symmetric, the Weingarten map is self-adjoint and the real eigenvalues of $\mathscr{W}$ are called principle curvatures, often denoted by $\lambda_{1}, \ldots, \lambda_{m}$, so that e.g. $H=\lambda_{1}+\cdots+\lambda_{m}$. Note, that in the theory of mean curvature flow $H$ is not the arithmetic means $\frac{1}{m} \sum_{i=1}^{m} \lambda_{i}$ (which would justify its name) as is often the case in classical books on differential geometry. In local coordinates we have

$$
A^{\alpha}{ }_{i j}=\nu^{\alpha} h_{i j}
$$

and then the equations of Gauß and Codazzi can be rewritten in terms of $h_{i j}$. E.g. since $|\nu|^{2}=1$ we have $\left\langle\nabla_{i} \nu, \nu\right\rangle=0$ and then

$$
\nabla_{i} \nu=\left\langle\nabla_{i} \nu, F^{m}\right\rangle F_{m}=-\left\langle\nu, \nabla_{i} F^{m}\right\rangle F_{m}=-h_{i}{ }^{m} F_{m} .
$$

This implies

$$
\begin{aligned}
\nabla_{i} A^{\alpha}{ }_{j k} & =\nabla_{i}\left(\nu^{\alpha} h_{j k}\right) \\
& =-h_{i}{ }^{m} h_{j k} F_{m}^{\alpha}+\nabla_{i} h_{j k} \nu^{\alpha} .
\end{aligned}
$$


Multiplying with $\nu_{\alpha}$ yields

$$
\left\langle\nabla_{i} A_{j k}, \nu\right\rangle=\nabla_{i} h_{j k}
$$

Interchanging $i, j$ and subtracting gives

$$
\begin{aligned}
\nabla_{i} h_{j k}-\nabla_{j} h_{i k} & =\left\langle\nabla_{i} A_{j k}-\nabla_{j} A_{i k}, \nu\right\rangle \\
& \stackrel{13}{=} R_{\alpha \beta \gamma \delta} \nu^{\alpha} F^{\beta}{ }_{k} F_{i}^{\gamma} F_{j}^{\delta}=R^{N}\left(\nu, F_{k}, F_{i}, F_{j}\right) .
\end{aligned}
$$

Similarly we get Gauß equation in the form

$$
R_{i j k l}=R^{N}\left(F_{i}, F_{j}, F_{k}, F_{l}\right)+h_{i k} h_{j l}-h_{i l} h_{j k}
$$

and since the codimension is one, we do not have a Ricci equation in this case.

\subsubsection{Lagrangian submanifolds.}

Let $(N, g=\langle\cdot, \cdot\rangle, J)$ be a Kähler manifold, i.e. $J \in \operatorname{End}(T N)$ is a parallel complex structure compatible with $g$. Then $N$ becomes a symplectic manifold with the symplectic form $\omega$ given by the Kähler form $\omega(V, W)=\langle J V, W\rangle$. An immersion $F: M \rightarrow N$ is called Lagrangian, if $F^{*} \omega=0$ and $n=\operatorname{dim} N=2 m=2 \operatorname{dim} M$. For a Lagrangian immersion we define a section

$$
\nu \in \Gamma\left(T^{\perp} M \otimes T^{*} M\right), \quad \nu:=J D F,
$$

where $J$ is applied to the $F^{*} T N$-part of $D F . \nu$ is a 1 -form with values in $T^{\perp} M$ since by the Lagrangian condition $J$ induces a bundle isomorphism (actually even a bundle isometry) between $D F(T M)$ and $T^{\perp} M$. In local coordinates $\nu$ can be written as

$$
\nu=\nu_{i} d x^{i}=\nu^{\alpha}{ }_{i} \frac{\partial}{\partial y^{\alpha}} \otimes d x^{i}
$$

with

$$
\nu_{i}=J F_{i}=J_{\beta}^{\alpha} F_{i}^{\beta} \frac{\partial}{\partial y^{\alpha}}, \quad \nu_{i}^{\alpha}=J_{\beta}^{\alpha} F_{i}^{\beta} .
$$

Since $J$ is parallel, we have

$$
\nabla \nu=J \nabla D F=J A
$$

In contrast to hypersurfaces, we may now define a second fundamental form as a tri-linear form

$$
h(X, Y, Z):=\langle\nu(X), A(Y, Z)\rangle .
$$

It turns out that $h$ is fully symmetric. Moreover, taking a trace, we obtain a 1 -form $H \in \Omega^{1}(M)$, called the mean curvature form,

$$
H(X):=\operatorname{trace} h(X, \cdot, \cdot) .
$$

In local coordinates

$$
h=h_{i j k} d x^{i} \otimes d x^{j} \otimes d x^{k}, \quad H=H_{i} d x^{i}, \quad H_{i}=g^{k l} h_{i k l} .
$$

The second fundamental tensor $A$ and the mean curvature vector $\vec{H}$ can be written in the form

$$
A^{\alpha}{ }_{i j}=h_{i j}{ }^{k} \nu_{k}^{\alpha}, \quad \vec{H}=H^{k} \nu_{k} .
$$

Since $J$ gives an isometry between the normal and tangent bundle of $M$, the equations of Gauß and Ricci coincide, so that we get the single equation

$$
R_{i j k l}=R^{N}\left(F_{i}, F_{j}, F_{k}, F_{l}\right)+h_{i k m} h_{j l}{ }^{m}-h_{i l m} h_{j k}{ }^{m} .
$$


Since $\nabla J=0$ and $J^{2}=-$ Id we also get

$$
\nabla_{i} \nu_{j}^{\alpha}=\nabla_{i}\left(J_{\beta}^{\alpha} F_{j}^{\beta}\right)=J_{\beta}^{\alpha} \nabla_{i} F_{j}^{\beta}=J_{\beta}^{\alpha} A_{i j}^{\beta}=J^{\alpha}{ }_{\beta} \nu_{k}^{\beta} h_{i j}{ }^{k}=-h_{i j}{ }^{k} F_{k}^{\alpha} .
$$

Similarly as above we conclude

$$
\begin{aligned}
& \nabla_{i} h_{j k l}-\nabla_{j} h_{i k l} \underset{\left(\nabla \nu_{l} \in D F(T M)\right)}{=} \nabla_{i}\left\langle A_{j k}, \nu_{l}\right\rangle-\nabla_{j}\left\langle A_{i k}, \nu_{l}\right\rangle \\
& \left(\nabla \nu_{l} \in D F(T M)\right) \quad\left\langle\nabla_{i} A_{j k}-\nabla_{j} A_{i k}, \nu_{l}\right\rangle \\
& \stackrel{13}{=} \quad R^{N}\left(\nu_{l}, F_{k}, F_{i}, F_{j}\right) \text {. }
\end{aligned}
$$

Taking a trace over $k$ and $l$, we deduce

$$
\nabla_{i} H_{j}-\nabla_{j} H_{i}=R^{N}\left(\nu_{k}, F^{k}, F_{i}, F_{j}\right)
$$

and if we take into account that $N$ is Kähler and $M$ Lagrangian, then the RHS is a Ricci curvature, so that the exterior derivative $d H$ of the mean curvature form $H$ is given by

$$
(d H)_{i j}=\nabla_{i} H_{j}-\nabla_{j} H_{i}=-\operatorname{Ric}^{N}\left(\nu_{i}, F_{j}\right)
$$

If $(N, g, J)$ is Kähler-Einstein, then $H$ is closed $\left(\operatorname{since~} \operatorname{Ric}^{N}\left(\nu_{i}, F_{j}\right)=c \cdot \omega\left(F_{i}, F_{j}\right)=\right.$ 0 ) and defines a cohomology class on $M$. In this case any (in general only locally defined) function $\alpha$ with $d \alpha=H$ is called a Lagrangian angle. In some sense the Lagrangian condition is an integrability condition. If we represent a Lagrangian submanifold locally as the graph over its tangent space, then the $m$ "height" functions are not completely independent but are related to a common potential. An easy way to see this, is to consider a locally defined 1-form $\lambda$ on $M$ (in a neighborhood of some point of $F(M)$ ) with $d \lambda=\omega$. Then by the Lagrangian condition

$$
0=F^{*} \omega=F^{*} d \lambda=d F^{*} \lambda .
$$

So $F^{*} \lambda$ is closed and by Poincaré's Lemma locally integrable. By the implicit function theorem this potential for $\lambda$ is related to the height functions of $M$ (cf. Smo00]). Note also that by a result of Weinstein for any Lagrangian embedding $M \subset N$ there exists a tubular neighborhood of $M$ which is symplectomorphic to $T^{*} M$ with its canonical symplectic structure $\omega=d \lambda$ induced by the Liouville form $\lambda$.

\subsubsection{Graphs.}

$\operatorname{Let}\left(M, g^{M}\right),\left(K, g^{K}\right)$ be two Riemannian manifolds and $f: M \rightarrow K$ a smooth map. $f$ induces a graph

$$
F: M \rightarrow N:=M \times K, \quad F(p):=(p, f(p)) .
$$

Since $N$ is also a Riemannian manifold equipped with the product metric $g=$ $g^{M} \times g^{K}$ one may consider the geometry of such graphs. It is clear that the geometry of $F$ must be completely determined by $f, g^{M}$ and $g^{K}$. Local coordinates $\left(x^{i}\right)_{i=1, \ldots, m},\left(z^{A}\right)_{A=1, \ldots, k}$ for $M$ resp. $K$ induce local coordinates $\left(y^{\alpha}\right)_{\alpha=1, \ldots, n=m+k}$ on $N$ by $y=(x, z)$. Then locally

$$
F_{i}(x)=\frac{\partial}{\partial x^{i}}+f_{i}^{A}(x) \frac{\partial}{\partial z^{A}}
$$


where similarly as before $f^{A}=z^{A} \circ f \circ x^{-1}$ and $f^{A}{ }_{i}=\frac{\partial f^{A}}{\partial x^{i}}$. For the induced metric $F^{*} g=g_{i j} d x^{i} \otimes d x^{j}$ we get

$$
g_{i j}=g_{i j}^{M}+g_{A B}^{K} f_{i}^{A} f_{j}^{B} .
$$

Since this is obviously positive definite and $F$ is injective, graphs $F: M \rightarrow M \times K$ of smooth mappings $f: M \rightarrow K$ are always embeddings. From the formula for $D F=F_{i} d x^{i}$ and the Gauß formula one may then compute the second fundamental tensor $A=\nabla D F$. Since the precise formula for $A$ is not important in this article, we leave the details as an exercise to the reader.

\section{General Results in higher CODimension}

In this section we focus on results in mean curvature flow that are valid in any dimension and codimension and that do not depend on specific geometric situations.

\subsection{Short-time existence and uniqueness.}

Consider the mean curvature vector field $\vec{H}=\vec{H}[F]$ as an operator on the class of smooth immersions

$$
\mathscr{I}:=\{F: M \rightarrow N: F \text { is a smooth immersion }\} .
$$

We want to compute the linearized operator belonging to $\vec{H}$. To this end we need to look at the symbol and therefore we consider the locally defined expression

$$
L_{\beta}^{\alpha ; i j}:=\frac{\partial H^{\alpha}}{\partial F_{i j}^{\beta}}
$$

where $F_{i j}^{\beta}$ is shorthand for $\frac{\partial^{2} F^{\beta}}{\partial x^{i} \partial x^{j}}$ and locally $\vec{H}=H^{\alpha} \frac{\partial}{\partial y^{\alpha}}$.

Let $g_{k i, j}:=\partial g_{k i} / \partial x^{j}$. We start with

$$
\begin{aligned}
\frac{\partial g_{k t, m}}{\partial F_{i j}^{\beta}} & =\frac{\partial}{\partial F_{i j}^{\beta}}\left(g_{\delta \epsilon, \rho} F_{k}^{\delta} F_{t}^{\epsilon} F^{\rho}{ }_{m}+g_{\delta \epsilon}\left(F_{k m}^{\delta} F_{t}^{\epsilon}+F^{\delta}{ }_{k} F_{t m}^{\epsilon}\right)\right) \\
& =g_{\beta \epsilon} \delta^{j}{ }_{m}\left(F_{t}^{\epsilon} \delta^{i}{ }_{k}+F^{\epsilon}{ }_{k} \delta^{i}{ }_{t}\right) .
\end{aligned}
$$

From this we then obtain

$$
\begin{aligned}
\frac{\partial \Gamma_{k m}^{s}}{\partial F_{i j}^{\beta}}= & \frac{1}{2} g^{s t} g_{\beta \epsilon}\left(\left(\delta^{i}{ }_{k} \delta^{j}{ }_{m}+\delta^{i}{ }_{m} \delta^{j}{ }_{k}\right) F^{\epsilon}{ }_{t}\right. \\
& \left.+\left(\delta^{i}{ }_{t}{ }^{j}{ }_{m}-\delta^{i}{ }_{m} \delta^{j}{ }_{t}\right) F^{\epsilon}{ }_{k}+\left(\delta^{i}{ }_{t} \delta^{j}{ }_{k}-\delta^{i}{ }_{k}{ }^{j}{ }_{t}\right) F^{\epsilon}{ }_{m}\right) .
\end{aligned}
$$

Since by Gauß' formula

$$
H^{\alpha}=g^{k m} A_{k m}^{\alpha}=g^{k m}\left(F_{k m}^{\alpha}-\Gamma_{k m}^{s} F_{s}^{\alpha}+\Gamma_{\beta \gamma}^{\alpha} F_{k}^{\beta} F_{m}^{\gamma}\right)
$$

we obtain

$$
\begin{aligned}
L_{\beta}^{\alpha ; i j}= & g^{k m}\left(\delta^{\alpha}{ }_{\beta} \delta^{i}{ }_{k} \delta^{j}{ }_{m}-\frac{1}{2} g^{s t} g_{\beta \epsilon}\left(\left(\delta^{i}{ }_{k} \delta^{j}{ }_{m}+\delta^{i}{ }_{m} \delta^{j}{ }_{k}\right) F^{\epsilon}{ }_{t}\right.\right. \\
& \left.\left.+\left(\delta^{i}{ }^{j}{ }^{j}{ }_{m}-\delta^{i}{ }_{m} \delta^{j}{ }_{t}\right) F^{\epsilon}{ }_{k}+\left(\delta^{i}{ } \delta^{j}{ }_{k}-\delta^{i}{ }_{k} \delta^{j}{ }_{t}\right) F^{\epsilon}{ }_{m}\right) F^{\alpha}{ }_{s}\right) \\
= & \delta^{\alpha}{ }_{\beta} g^{i j}-g^{s t}{ }_{\beta}{ }_{\beta \epsilon} g^{i j} F_{t}^{\epsilon} F^{\alpha}{ }_{s}-\left(g^{k j} g^{s i}-g^{k i} g^{s j}\right) g_{\beta \epsilon} F^{\epsilon}{ }_{k} F^{\alpha}{ }_{s} .
\end{aligned}
$$


For an arbitrary nonzero 1-form $\xi=\xi_{i} d x^{i}$ we define the endomorphism $L=$ $\left(L_{\beta}^{\alpha}\right)_{\alpha, \beta=1, \ldots, n}$ by

$$
L_{\beta}^{\alpha}:=L_{\beta}^{\alpha ; i j} \xi_{i} \xi_{j} .
$$

We compute

$$
L_{\beta}^{\alpha}=\left(\delta_{\beta}^{\alpha}-g_{\beta \epsilon} g^{s t} F_{t}^{\epsilon} F_{s}^{\alpha}\right)|\xi|^{2} .
$$

Applying this to a tangent vector $F_{l}=F^{\beta}{ }_{l} \frac{\partial}{\partial y^{\beta}}$ we get

$$
L^{\alpha}{ }_{\beta} F_{l}^{\beta}=0 .
$$

If $\nu=\nu^{\beta} \frac{\partial}{\partial y^{\beta}}$ is normal, then

$$
g_{\beta \epsilon} \nu^{\beta} F_{t}^{\epsilon}=0
$$

and hence

$$
L^{\alpha}{ }_{\beta} \nu^{\beta}=|\xi|^{2} \nu^{\alpha} .
$$

Consequently $L$ is degenerate along tangent directions of $F$ and elliptic along normal directions, more precisely for $\xi \in T_{p}^{*} M$ we have

$$
L_{\mid p}=|\xi|^{2} \pi_{\mid p},
$$

where $\pi_{\mid p}: T_{F(p)} N \rightarrow T_{p}^{\perp} M$ is the projection of $T_{F(p)} N$ onto $T_{p}^{\perp} M$. The reason for the $m$ degeneracies is the following: Writing a solution $F: M \rightarrow N$ of $\vec{H}=0$ locally as the graph over its tangent plane at $F(p)$, we see that we need as many height functions as there are codimensions, i.e. we need $k=n-m$ functions. On the other hand the system $H^{\alpha}=0, \alpha=1, \ldots, n$ consists of $n$ coupled equations and is therefore overdetermined with a redundancy of $m$ equations. These $m$ redundant equations correspond to the diffeomorphism group of the underlying $m$-dimensional manifold $M$. This means the following:

Proposition 3.1 (Invariance under the diffeomorphism group). If $F: M \times[0, T) \rightarrow$ $N$ is a solution of the mean curvature flow, and $\phi \in \operatorname{Diff}(M)$ a fixed diffeomorphism of $M$, then $\tilde{F}: M \times[0, T) \rightarrow N, \tilde{F}(p, t):=F(\phi(p), t)$ is another solution. In particular, the (immersed) submanifolds $\tilde{M}_{t}:=\tilde{F}(M, t)$ and $M_{t}:=F(M, t)$ coincide for all $t$.

Thus the mean curvature flow is a (degenerate) quasilinear parabolic evolution equation. The following theorem is well-known and in particular forms a special case of a theorem by Richard Hamilton Ham82b, based on the Nash-Moser implicit function theorem treated in another paper by Hamilton Ham82a.

Proposition 3.2 (Short-time existence and uniqueness). Let $M$ be a smooth closed manifold and $F_{0}: M \rightarrow N$ a smooth immersion into a smooth Riemannian manifold $(N, g)$. Then the mean curvature flow admits a unique smooth solution on a maximal time interval $[0, T), 0<T \leq \infty$.

Besides the invariance of the equation under the diffeomorphism group of $M$ the flow is isotropic, i.e. invariant under isometries of the ambient space. This property follows from the invariance of the first and second fundamental forms under isometries. 
Proposition 3.3 (Invariance under isometries). Suppose $F: M \times[0, T) \rightarrow N$ is a smooth solution of the mean curvature flow and assume that $\phi$ is an isometry of the ambient space $(N, g)$. Then the family $\tilde{F}:=\phi \circ F$ is another smooth solution of the mean curvature flow. In particular, if the initial immersion is invariant under $\phi$, then it will stay invariant for all $t \in[0, T)$.

We note that the short-time existence and uniqueness result stated above is not in the most general form. For example, it is not necessary to assume smoothness initially, it suffices to assume Lipschitz continuity. We note also that in general the short-time existence and uniqueness result for non-compact complete manifolds $M$ is open but there exist important contributions in special cases. Based on interior estimates, Ecker and Huisken EH91 proved - requiring only a local Lipschitz condition for the initial hypersurface -, a short-time existence result for the mean curvature flow of complete hypersurfaces. In that paper the authors also show that the mean curvature flow smoothes out Lipschitz hypersurfaces (i.e. the solution becomes smooth for $t>0$ ). This short-time existence result has been improved in a paper by Colding and Minicozzi CM04 where one only needs to assume a local bound for the initial height function. The smoothing out result by Ecker and Huisken has been extended by Wang to any dimension and codimension in Wan04 provided the submanifolds have a small local Lipschitz norm (which cannot be improved by an example of Lawson and Osserman) and the ambient space has bounded geometry. Recently Chen and Yin CY07 proved that uniqueness for complete manifolds $M$ still holds within the class of smooth solutions with bounded second fundamental tensor, if the ambient Riemannian manifold $(N, g)$ has bounded geometry in a certain sense. Chen and Pang [CP09] considered uniqueness of unbounded solutions of the Lagrangian mean curvature flow equation for graphs.

\subsection{Evolution equations.}

Suppose $F: M \times[0, T) \rightarrow N$ is a smooth solution of the mean curvature flow

$$
\frac{d}{d t} F=\vec{H}
$$

In this subsection we want to state and prove evolution equations of the most important geometric quantities on $M$, like the first and second fundamental forms.

To this end we will compute evolution equations for various sections $\sigma$ in vector bundles $E$ over $M$. We will use the index notation introduced in subsection 2.3 In particular, we will consider those cases, where $\sigma$ is a section in a vector bundle $E_{t}$ which itself depends on time $t$. If for example $\nu_{t}$ is the principal normal vector field of a hypersurface $F: M \rightarrow N$, then $\nu_{t}$ is a section in $E_{t}:=F_{t}^{*} T N$. In this case the mere computation of the total derivative of $\nu_{t}$ w.r.t. $t$ will be insufficient since this would only make sense in local coordinates (local in space and time). To overcome this difficulty we just need to define a connection $\nabla$ on $F^{*} T N$, where $F$ is now considered as a smooth map (in general no immersion) from the space-time manifold $M \times[0, T)$ to $N$. A time derivative then becomes a covariant derivative in direction of $\frac{d}{d t}$, for example for a time dependent section $\nu \in F^{*} T N$ we have in local coordinates

$$
\nu(x, t)=\nu^{\alpha}(x, t) \frac{\partial}{\partial y^{\alpha}}
$$




$$
\nabla_{\frac{d}{d t}} \nu=\left(\frac{d \nu^{\alpha}}{d t}+\Gamma_{\beta \delta}^{\alpha} \frac{d F^{\beta}}{d t} \nu^{\delta}\right) \frac{\partial}{\partial y^{\alpha}}=\left(\frac{d \nu^{\alpha}}{d t}+\Gamma_{\beta \delta}^{\alpha} H^{\beta} \nu^{\delta}\right) \frac{\partial}{\partial y^{\alpha}},
$$

where $\Gamma_{\beta \delta}^{\alpha}$ are the Christoffel symbols of the Levi-Civita connection on $N$ and $\left(y^{\alpha}\right)$ are local coordinates on $N$. On the other hand, if $\sigma$ is a section in a bundle $E$ and $E$ does not depend on $t$, then the covariant derivative $\nabla_{\frac{d}{d t}} \sigma$ coincides with $\frac{d}{d t} \sigma$. For example for the induced metric $F_{t}^{*} g \in \Gamma\left(T^{*} M \otimes T^{*} M\right)$ we have

$$
F_{t}^{*} g=g_{i j}(x, t) d x^{i} \otimes d x^{j}
$$

and

$$
\nabla_{\frac{d}{d t}} F_{t}^{*} g=\frac{d}{d t} g_{i j}(x, t) d x^{i} \otimes d x^{j}
$$

since $T^{*} M$ does not depend on $t$. Likewise, for the second fundamental tensor $A$ (considered as a section in $F^{*} T N \otimes T^{*} M \otimes T^{*} M$, which makes sense since for $\tilde{M}=M \times[0, T)$ we have $\left.T^{*} \tilde{M}=T^{*} M \oplus T^{*} \mathbb{R}\right)$ we get

$$
\nabla_{\frac{d}{d t}} A^{\alpha}{ }_{i j}=\frac{d}{d t} A^{\alpha}{ }_{i j}+\Gamma_{\beta \gamma}^{\alpha} \frac{d F^{\beta}}{d t} A^{\gamma}{ }_{i j}=\frac{d}{d t} A^{\alpha}{ }_{i j}+\Gamma_{\beta \gamma}^{\alpha} H^{\beta} A^{\gamma}{ }_{i j} .
$$

Lemma 3.4. If $F: M \times[0, T) \rightarrow(N, g)$ evolves under the mean curvature flow, then the induced Riemannian metrics $F_{t}^{*} g=g_{i j}(x, t) d x^{i} \otimes d x^{j} \in \Gamma\left(T^{*} M \otimes T^{*} M\right)$ evolve according to

$$
\nabla_{\frac{d}{d t}} g_{i j}=\frac{d}{d t} g_{i j}=-2\left\langle\vec{H}, A_{i j}\right\rangle
$$

Proof. We have

and thus

$$
g_{i j}=g_{\alpha \beta} F_{i}^{\alpha} F_{j}^{\beta}
$$

$$
\begin{aligned}
\nabla_{\frac{d}{d t}} g_{i j} & =\underbrace{\nabla_{\gamma} g_{\alpha \beta}}_{=0} \frac{d F^{\gamma}}{d t} F_{i}^{\alpha} F^{\beta}{ }_{j}+g_{\alpha \beta}\left(\nabla_{\frac{d}{d t}} F_{i}^{\alpha} F_{j}^{\beta}+F_{i}^{\alpha} \nabla_{\frac{d}{d t}} F_{j}^{\beta}\right) \\
& =g_{\alpha \beta}\left(\nabla_{i} \frac{d F^{\alpha}}{d t} F^{\beta}{ }_{j}+F^{\alpha}{ }_{i} \nabla_{j} \frac{d F^{\beta}}{d t}\right) \\
& =g_{\alpha \beta}\left(\nabla_{i} H^{\alpha} F^{\beta}{ }_{j}+F^{\alpha}{ }_{i} \nabla_{j} H^{\beta}\right),
\end{aligned}
$$

where we have used that $\nabla_{\gamma} g_{\alpha \beta}=0$ (since $\nabla$ is metric) and $\nabla_{\frac{d}{d t}} F_{i}^{\alpha}=\nabla_{i} \frac{d F^{\alpha}}{d t}$. This last identity holds since the second fundamental tensor $\tilde{A} \in \Gamma\left(F^{*} T N \otimes T^{*} \tilde{M} \otimes T^{*} \tilde{M}\right)$ of the map $F: \tilde{M} \rightarrow N$ is symmetric, so that

$$
\tilde{A}\left(\frac{\partial}{\partial x^{i}}, \frac{d}{d t}\right)=\nabla_{i} \frac{d F^{\alpha}}{d t} \frac{\partial}{\partial y^{\alpha}}=\nabla_{\frac{d}{d t}} F_{i}^{\alpha} \frac{\partial}{\partial y^{\alpha}}=\tilde{A}\left(\frac{d}{d t}, \frac{\partial}{\partial x^{i}}\right) .
$$

Now since $g_{\alpha \beta} H^{\alpha} F^{\beta}{ }_{j}=0$, we get

$$
\begin{aligned}
0 & =\nabla_{i}\left(g_{\alpha \beta} H^{\alpha} F^{\beta}{ }_{j}\right) \\
& =\nabla_{\gamma} g_{\alpha \beta} F^{\gamma}{ }_{i} H^{\alpha} F^{\beta}{ }_{j}+g_{\alpha \beta}\left(\nabla_{i} H^{\alpha} F^{\beta}{ }_{j}+H^{\alpha} \nabla_{i} F^{\beta}{ }_{j}\right) \\
& =g_{\alpha \beta}\left(\nabla_{i} H^{\alpha} F^{\beta}{ }_{j}+H^{\alpha} A^{\beta}{ }_{i j}\right)
\end{aligned}
$$

since $\nabla_{i} F_{j}^{\beta}=A^{\beta}{ }_{i j}$. If we insert this into equation (19), then we obtain the result. 
Corollary 3.5. The induced volume form $d \mu_{t}$ on $M$ evolves according to

$$
\nabla_{\frac{d}{d t}} d \mu_{t}=\frac{d}{d t} d \mu_{t}=-|\vec{H}|^{2} d \mu_{t} .
$$

Proof. In local coordinates we have

$$
d \mu_{t}=\sqrt{\operatorname{det} g_{k l}} d x^{1} \wedge \cdots \wedge d x^{m} .
$$

Since

$$
\frac{d}{d t}\left(\operatorname{det} g_{k l}\right)=\left(g^{i j} \frac{d}{d t} g_{i j}\right) \operatorname{det} g_{k l}
$$

the claim follows easily.

Corollary 3.6. The Christoffel symbols $\Gamma_{i j}^{k}$ of the Levi-Civita connection on $M$ evolve according to

$$
\frac{d}{d t} \Gamma_{i j}^{k}=-g^{k l}\left(\nabla_{i}\left\langle\vec{H}, A_{j l}\right\rangle+\nabla_{j}\left\langle\vec{H}, A_{i l}\right\rangle-\nabla_{l}\left\langle\vec{H}, A_{i j}\right\rangle\right) .
$$

Proof. This follows directly from

$$
\Gamma_{i j}^{k}=\frac{1}{2} g^{k l}\left(g_{i l, j}+g_{j l, i}-g_{i j, l}\right),
$$

the evolution equation of the metric and the fact that $\frac{d}{d t} \Gamma_{i j}^{k}$ is a tensor (though $\Gamma_{i j}^{k}$ is not).

Next we compute the evolution equation for the second fundamental tensor $A=$ $A^{\alpha}{ }_{i j} \frac{\partial}{\partial y^{\alpha}} \otimes d x^{i} \otimes d x^{j}$

Lemma 3.7. The second fundamental tensor $A$ evolves under the mean curvature flow by

$$
\nabla_{\frac{d}{d t}} A^{\alpha}{ }_{i j}=\nabla_{i} \nabla_{j} H^{\alpha}-C_{i j}^{k} F_{k}^{\alpha}+R_{\delta \gamma \epsilon}^{\alpha} F_{j}^{\delta} H^{\gamma} F_{i}^{\epsilon},
$$

where $C_{i j}^{k}=\frac{d}{d t} \Gamma_{i j}^{k}$.

Proof. Since

$$
A^{\alpha}{ }_{i j}=\frac{\partial^{2} F^{\alpha}}{\partial x^{i} \partial x^{j}}-\Gamma_{i j}^{k} F_{k}^{\alpha}+\Gamma_{\beta \gamma}^{\alpha} F_{i}^{\beta} F_{j}^{\gamma}
$$

we get

$$
\begin{aligned}
\frac{d}{d t} A_{i j}^{\alpha}= & \frac{\partial^{2} H^{\alpha}}{\partial x^{i} \partial x^{j}}-\Gamma_{i j}^{k} \frac{\partial H^{\alpha}}{\partial x^{k}}+\Gamma_{\beta \gamma}^{\alpha}\left(\frac{\partial H^{\beta}}{\partial x^{i}} F^{\gamma}{ }_{j}+F^{\beta}{ }_{i} \frac{\partial H^{\gamma}}{\partial x^{j}}\right) \\
& -\frac{d}{d t} \Gamma_{i j}^{k} F_{k}^{\alpha}+\Gamma_{\beta \gamma, \delta}^{\alpha} H^{\delta} F^{\beta}{ }_{i} F_{j}^{\gamma} .
\end{aligned}
$$

To continue we need some covariant expressions. For a section $V=V^{\alpha} \frac{\partial}{\partial y^{\alpha}} \in$ $\Gamma\left(F^{*} T N\right)$ we have

$$
\nabla_{j} V^{\alpha}=\frac{\partial V^{\alpha}}{\partial x^{j}}+\Gamma_{\beta \gamma}^{\alpha} F_{j}^{\beta} V^{\gamma}
$$


and then

$$
\begin{aligned}
\nabla_{i} \nabla_{j} V^{\alpha}= & \frac{\partial}{\partial x^{i}}\left(\frac{\partial V^{\alpha}}{\partial x^{j}}+\Gamma_{\beta \gamma}^{\alpha} F_{j}^{\beta} V^{\gamma}\right)-\Gamma_{i j}^{k}\left(\frac{\partial V^{\alpha}}{\partial x^{k}}+\Gamma_{\beta \gamma}^{\alpha} F_{k}^{\beta} V^{\gamma}\right) \\
& +\Gamma_{\beta \gamma}^{\alpha} F^{\beta}{ }_{i}\left(\frac{\partial V^{\gamma}}{\partial x^{j}}+\Gamma_{\delta \epsilon}^{\gamma} F^{\delta}{ }_{j} V^{\epsilon}\right) \\
= & \frac{\partial^{2} V^{\alpha}}{\partial x^{i} \partial x^{j}}+\Gamma_{\beta \gamma, \delta}^{\alpha} F_{i}^{\delta} F^{\beta}{ }_{j} V^{\gamma}+\Gamma_{\beta \gamma}^{\alpha} \frac{\partial^{2} F^{\beta}}{\partial x^{i} \partial x^{j}} V^{\gamma}+\Gamma_{\beta \gamma}^{\alpha} F^{\beta}{ }_{j} \frac{\partial V^{\gamma}}{\partial x^{i}} \\
& -\Gamma_{i j}^{k}\left(\frac{\partial V^{\alpha}}{\partial x^{k}}+\Gamma_{\beta \gamma}^{\alpha} F^{\beta}{ }_{k} V^{\gamma}\right)+\Gamma_{\beta \gamma}^{\alpha} F^{\beta}{ }_{i}\left(\frac{\partial V^{\gamma}}{\partial x^{j}}+\Gamma_{\delta \epsilon}^{\gamma} F_{j}^{\delta} V^{\epsilon}\right) \\
= & \frac{\partial^{2} V^{\alpha}}{\partial x^{i} \partial x^{j}}-\Gamma_{i j}^{k} \frac{\partial V^{\alpha}}{\partial x^{k}}+\Gamma_{\beta \gamma}^{\alpha}\left(\frac{\partial V^{\beta}}{\partial x^{i}} F^{\gamma}{ }_{j}+F^{\beta}{ }_{i} \frac{\partial V^{\gamma}}{\partial x^{j}}\right) \\
& +\Gamma_{\beta \gamma}^{\alpha} \frac{\partial^{2} F^{\beta}}{\partial x^{i} \partial x^{j}} V^{\gamma}-\Gamma_{i j}^{k} \Gamma_{\beta \gamma}^{\alpha} F^{\beta}{ }_{k} V^{\gamma}+\Gamma_{\beta \epsilon}^{\alpha} \Gamma_{\delta \gamma}^{\beta} F_{i}^{\epsilon} F^{\delta}{ }_{j} V^{\gamma} \\
& +\Gamma_{\beta \gamma, \delta}^{\alpha} F_{i}^{\delta} F^{\beta}{ }_{j} V^{\gamma} \\
= & \frac{\partial^{2} V^{\alpha}}{\partial x^{i} \partial x^{j}}-\Gamma_{i j}^{k} \frac{\partial V^{\alpha}}{\partial x^{k}}+\Gamma_{\beta \gamma}^{\alpha}\left(\frac{\partial V^{\beta}}{\partial x^{i}} F^{\gamma}{ }_{j}+F^{\beta}{ }_{i} \frac{\partial V^{\gamma}}{\partial x^{j}}\right) \\
& +\Gamma_{\beta \gamma}^{\alpha} V^{\gamma} A^{\beta}{ }_{i j}+\left(\Gamma_{\beta \epsilon}^{\alpha} \Gamma_{\delta \gamma}^{\beta}-\Gamma_{\beta \gamma}^{\alpha} \Gamma_{\delta \epsilon}^{\beta}\right) F_{i}^{\epsilon} F_{j}^{\delta}{ }_{j}^{\gamma}+\Gamma_{\beta \gamma, \delta}^{\alpha} F_{i}^{\delta} F_{j}^{\beta} V^{\gamma},
\end{aligned}
$$

where we have used $\Gamma_{\beta \gamma}^{\alpha}=\Gamma_{\gamma \beta}^{\alpha}$ several times.

Applying this to $V^{\alpha}=H^{\alpha}$ we conclude

$$
\begin{aligned}
\frac{d}{d t} A^{\alpha}{ }_{i j}= & \nabla_{i} \nabla_{j} H^{\alpha}-\frac{d}{d t} \Gamma_{i j}^{k} F_{k}^{\alpha}+\Gamma_{\beta \gamma, \delta}^{\alpha} H^{\delta} F_{i}^{\beta} F_{j}^{\gamma} \\
& -\Gamma_{\beta \gamma}^{\alpha} H^{\gamma} A^{\beta}{ }_{i j}-\left(\Gamma_{\beta \epsilon}^{\alpha} \Gamma_{\delta \gamma}^{\beta}-\Gamma_{\beta \gamma}^{\alpha} \Gamma_{\delta \epsilon}^{\beta}\right) F_{i}^{\epsilon} F_{j}^{\delta} H^{\gamma}-\Gamma_{\beta \gamma, \delta}^{\alpha} F_{i}^{\delta} F^{\beta}{ }_{j} H^{\gamma} \\
= & \nabla_{i} \nabla_{j} H^{\alpha}-\frac{d}{d t} \Gamma_{i j}^{k} F_{k}^{\alpha}-\Gamma_{\beta \gamma}^{\alpha} H^{\gamma} A^{\beta}{ }_{i j} \\
& +\left(\Gamma_{\epsilon \delta, \gamma}^{\alpha}-\Gamma_{\gamma \delta, \epsilon}^{\alpha}-\Gamma_{\beta \epsilon}^{\alpha} \Gamma_{\delta \gamma}^{\beta}+\Gamma_{\beta \gamma}^{\alpha} \Gamma_{\delta \epsilon}^{\beta}\right) F_{i}^{\epsilon} F_{j}^{\delta} H^{\gamma} \\
= & \nabla_{i} \nabla_{j} H^{\alpha}-\frac{d}{d t} \Gamma_{i j}^{k} F_{k}^{\alpha}-\Gamma_{\beta \gamma}^{\alpha} H^{\gamma} A^{\beta}{ }_{i j}+R_{\delta \gamma \epsilon}^{\alpha} F_{i}{ }_{i} F_{j}^{\delta} H^{\gamma} .
\end{aligned}
$$

The result then follows from (17).

Corollary 3.8. Under the mean curvature flow the mean curvature satisfies the following evolution equations:

$$
\begin{aligned}
\nabla_{\frac{d}{d t}} H^{\alpha}= & \Delta H^{\alpha}-g^{i j} C_{i j}^{k} F_{k}^{\alpha}+R_{\delta \gamma \epsilon}^{\alpha} F_{i}^{\epsilon} F^{\delta i} H^{\gamma}+2\left\langle A_{k l}, \vec{H}\right\rangle A^{\alpha k l} \\
\nabla_{\frac{d}{d t}}|\vec{H}|^{2}= & \Delta|\vec{H}|^{2}-2|\nabla \vec{H}|^{2}+4\left\langle A^{i j}, \vec{H}\right\rangle\left\langle A_{i j}, \vec{H}\right\rangle \\
& +2 R_{\alpha \beta \gamma \delta} H^{\alpha} F^{\beta} H_{i}^{\gamma} F^{\delta i} \\
= & \Delta|\vec{H}|^{2}-2\left|\nabla^{\perp} \vec{H}\right|^{2}+2\left\langle A^{i j}, \vec{H}\right\rangle\left\langle A_{i j}, \vec{H}\right\rangle \\
& +2 R_{\alpha \beta \gamma \delta} H^{\alpha} F_{i}^{\beta} H^{\gamma} F^{\delta i}
\end{aligned}
$$

Proof. The first equation follows from $H^{\alpha}=g^{i j} A^{\alpha}{ }_{i j}$, equations (18), (22) and

$$
\nabla_{\frac{d}{d t}} g^{i j}=-g^{i k} g^{j l} \nabla_{\frac{d}{d t}} g_{k l}
$$


The second equation then follows from $|\vec{H}|^{2}=g_{\alpha \beta} H^{\alpha} H^{\beta}, g_{\alpha \beta} F_{i}^{\alpha} H^{\beta}=0$ and

$$
\nabla_{\frac{d}{d t}} g_{\alpha \beta}=\nabla_{\gamma} g_{\alpha \beta} H^{\gamma}=0 \text {. }
$$

Finally, (26) follows from

$$
\begin{aligned}
\nabla_{k} \vec{H} & =\nabla_{k}^{\perp} \vec{H}+g^{i j}\left\langle\nabla_{k} \vec{H}, F_{i}\right\rangle F_{j} \\
& =\nabla_{k}^{\perp} \vec{H}-g^{i j}\left\langle\vec{H}, \nabla_{k} F_{i}\right\rangle F_{j} \\
& =\nabla_{k}^{\perp} \vec{H}-g^{i j}\left\langle\vec{H}, A_{k i}\right\rangle F_{j}
\end{aligned}
$$

and $\left\langle\nabla_{k}^{\perp} \vec{H}, F_{j}\right\rangle=0$.

From the evolution equation of $A^{\alpha}{ }_{i j}$ we obtain in the same way

$$
\begin{aligned}
\nabla_{\frac{d}{d t}}|A|^{2}= & 2\left\langle\nabla^{2} \vec{H}, A\right\rangle+4\left\langle\vec{H}, A^{i j}\right\rangle\left\langle A_{i k}, A_{j}{ }^{k}\right\rangle \\
& +2 R_{\alpha \beta \gamma \delta} A^{\alpha k l} F^{\beta}{ }_{k} H^{\gamma} F^{\delta}{ }_{l}
\end{aligned}
$$

Applying Simons' identity (16) we get

$$
\begin{aligned}
\nabla_{\frac{d}{d t}}|A|^{2}= & \Delta|A|^{2}-2\left|\nabla^{\perp} A\right|^{2} \\
& +\left|\left\langle A_{i j}, A_{k l}\right\rangle-\left\langle A_{i l}, A_{j k}\right\rangle\right|^{2}+\left|A^{\alpha}{ }_{i k} A^{\beta}{ }_{j}{ }^{2}-A^{\beta}{ }_{i k} A^{\alpha}{ }_{j}{ }^{k}\right|^{2} \\
& +2\left|\left\langle\vec{H}, A_{i j}\right\rangle-\left\langle A_{i k}, A_{j}{ }^{k}\right\rangle\right|^{2}-2\left|\left\langle\vec{H}, A_{i j}\right\rangle\right|^{2} \\
& +4 R_{\alpha \beta \gamma \delta} F^{\alpha}{ }_{k} F^{\beta}{ }_{i} F^{\gamma} F^{\delta}{ }_{j}\left(\left\langle A^{i j}, A^{k l}\right\rangle-g^{k l}\left\langle A^{i p}, A_{p}{ }^{j}\right\rangle\right) \\
& +2 R_{\alpha \beta \gamma \delta} A^{\alpha k l}\left(4 A^{\beta}{ }_{i k} F^{\gamma}{ }_{l} F^{\delta i}+F^{\beta}{ }_{l} F^{\gamma}{ }_{k} H^{\delta}+F^{\beta}{ }_{i} A^{\gamma}{ }_{l k} F^{\delta i}\right) \\
& +2\left(\nabla_{\epsilon} R_{\alpha \beta \gamma \delta}+\nabla_{\gamma} R_{\alpha \delta \beta \epsilon}\right) F_{i}{ }_{i} F^{\beta}{ }_{l} F^{\gamma}{ }_{k} F^{\delta i} A^{\alpha k l} \\
& +4\left\langle\vec{H}, A^{i j}\right\rangle\left\langle A_{i k}, A_{j}{ }^{k}\right\rangle \\
& +2 R_{\alpha \beta \gamma \delta} A^{\alpha k l} F^{\beta}{ }_{k} H^{\gamma} F^{\delta}{ }_{l} \\
= & \Delta|A|^{2}-2\left|\nabla^{\perp} A\right|^{2} \\
& +2\left|\left\langle A_{i j}, A_{k l}\right\rangle\right|^{2}+\left|A^{\alpha}{ }_{i k} A^{\beta}{ }_{j}{ }^{k}-A^{\beta}{ }_{i k} A^{\alpha}{ }_{j}{ }^{k}\right|^{2} \\
& +4 R_{\alpha \beta \gamma \delta} F^{\alpha}{ }_{k} F_{i}{ }_{i} F_{l}^{\gamma} F^{\delta}{ }_{j}\left(\left\langle A^{i j}, A^{k l}\right\rangle-g^{k l}\left\langle A^{i p}, A_{p}{ }^{j}\right\rangle\right) \\
& +2 R_{\alpha \beta \gamma \delta} A^{\alpha k l}\left(4 A^{\beta}{ }_{i k} F^{\gamma}{ }_{l} F^{\delta i}+F^{\beta}{ }_{i} A^{\gamma}{ }_{l k} F^{\delta i}\right) \\
& +2\left(\nabla_{\epsilon} R_{\alpha \beta \gamma \delta}+\nabla_{\gamma} R_{\alpha \delta \beta \epsilon}\right) F_{i}^{\epsilon} F^{\beta}{ }_{l} F^{\gamma}{ }_{k} F^{\delta i} A^{\alpha k l}
\end{aligned}
$$

Thus we have shown

Corollary 3.9. Under the mean curvature flow the quantity $|A|^{2}$ satisfies the following evolution equation:

$$
\begin{aligned}
\nabla_{\frac{d}{d t}}|A|^{2}= & \Delta|A|^{2}-2\left|\nabla^{\perp} A\right|^{2} \\
& +2\left|\left\langle A_{i j}, A_{k l}\right\rangle\right|^{2}+\left|A^{\alpha}{ }_{i k} A^{\beta}{ }_{j}{ }^{k}-A^{\beta}{ }_{i k} A^{\alpha}{ }_{j}{ }^{k}\right|^{2} \\
& +4 R_{\alpha \beta \gamma \delta} F^{\alpha}{ }_{k} F^{\beta}{ }_{i} F^{\gamma}{ }_{l} F^{\delta}{ }_{j}\left(\left\langle A^{i j}, A^{k l}\right\rangle-g^{k l}\left\langle A^{i p}, A_{p}{ }^{j}\right\rangle\right) \\
& +2 R_{\alpha \beta \gamma \delta} A^{\alpha k l}\left(4 A^{\beta}{ }_{i k} F^{\gamma}{ }_{l} F^{\delta i}+F^{\beta}{ }_{i} A^{\gamma}{ }_{l k} F^{\delta i}\right) \\
& +2\left(\nabla_{\epsilon} R_{\alpha \beta \gamma \delta}+\nabla_{\gamma} R_{\alpha \delta \beta \epsilon}\right) F^{\epsilon}{ }_{i} F^{\beta}{ }_{l} F^{\gamma}{ }_{k} F^{\delta i} A^{\alpha k l}
\end{aligned}
$$


These general evolution equations simplify in more special geometric situations. E.g., if the codimension is one, then $A^{\alpha}{ }_{i j}=\nu^{\alpha} h_{i j}$ (cf. subsection 2.4.1) implies $\left|\nabla^{\perp} A\right|^{2}=|\nabla h|^{2},|A|^{2}=|h|^{2}$ and

$$
\begin{aligned}
\nabla_{\frac{d}{d t}}|h|^{2}= & \Delta|h|^{2}-2|\nabla h|^{2}+2|h|^{2}\left(|h|^{2}+\overline{\operatorname{Ric}}(\nu, \nu)\right) \\
& -4\left(h^{i j} h_{j}{ }^{m} \bar{R}_{m l i}{ }^{l}-h^{i j} h^{l m} \bar{R}_{m i l j}\right) \\
& +2 h^{i j}\left(\bar{\nabla}_{j} \bar{R}_{0 l i}{ }^{l}+\bar{\nabla}_{l} \bar{R}_{0 i j}{ }^{l}\right)
\end{aligned}
$$

where

$$
\bar{R}_{m i l j}:=R_{\alpha \beta \gamma \delta} F_{m}^{\alpha} F_{i}^{\beta} F_{l}^{\gamma} F_{j}^{\delta}, \quad \overline{\operatorname{Ric}}(\nu, \nu):=R_{\alpha \beta \gamma \delta} \nu^{\alpha} F_{i}^{\beta} \nu^{\gamma} F^{\delta i}
$$

and

$$
\bar{\nabla}_{l} \bar{R}_{0 i j}{ }^{l}:=\nabla_{\alpha} R_{\beta \gamma \delta \epsilon} F_{l}^{\alpha} \nu^{\beta} F_{i}^{\gamma} F_{j}^{\delta} F^{\epsilon l} .
$$

Equation (29) is Corollary 3.5 (ii) in [Hui86]. Note that there is a plus sign in the last line of (29) since our unit normal is inward pointing and the unit normal in Hui86 is outward directed.

\subsection{Long-time existence.}

In general long-time existence of solutions cannot be expected as the following well-known theorem shows:

Proposition 3.10. Suppose $F_{0}: M \rightarrow \mathbb{R}^{n}$ is a smooth immersion of a closed $m$-dimensional manifold $M$. Then the maximal time $T$ of existence of a smooth solution $F: M \times[0, T) \rightarrow \mathbb{R}^{n}$ of the mean curvature flow with initial immersion $F_{0}$ is finite.

Proof. The proof easily follows by applying the parabolic maximum principle to the function $f:=|F|^{2}+2 m t$ which satisfies the evolution equation

$$
\frac{d}{d t} f=\Delta f
$$

Hence $T \leq \frac{1}{2 m} \max \left|F_{0}\right|^{2}$ and the inequality is sharp since equality is attained for round spheres centered at the origin.

This result is no longer true for complete submanifolds since for example for entire $m$-dimensional graphs in $\mathbb{R}^{m+1}$ one has long-time existence (see [EH89]). In addition, the result can fail, if the ambient space is a Riemannian manifold since in some cases one gets long-time existence and convergence (for example in Gra89, Smo02,Smo04, [SW02, Wan02], TW04]).

The next well known theorem holds in any case:

Proposition 3.11. Let $M$ be a closed manifold and $F: M \times[0, T) \rightarrow(N, g)$ a smooth solution of the mean curvature flow in a complete (compact or non-compact) Riemannian manifold $(N, g)$. Suppose the maximal time of existence $T$ is finite. Then

$$
\limsup _{t \rightarrow T} \max _{M_{t}}|A|^{2}=\infty .
$$

Here, $M_{t}:=F(M, t)$. 
Remark 3.12. The same result also holds in some other situations. For example one can easily see that under suitable assumptions on the solution one can allow $N$ to have boundary.

Proof. The theorem is one of the "folklore" results in mean curvature flow for which a rigorous proof in all dimensions and codimensions has not been written up in detail but can be carried out in the same way as the corresponding proof for hypersurfaces. This has been done by Huisken in Hui84, Hui90 and is again based on the maximum principle. The key observation is, that all higher derivatives $\nabla^{k} A$ of the second fundamental tensor are uniformly bounded, once $A$ is uniformly bounded. This can be shown by induction and has originally been carried out for hypersurfaces using $L^{p}$-estimates in Hui84. For compact hypersurfaces there exists a more direct argument involving the maximum principle applied to the evolution equations of $|A|^{2}$ in (28) and $\left|\nabla^{k} A\right|^{2}$. The method can be found in the proof of Proposition 2.3 in Hui90 and works in the same way in any codimension and in any ambient Riemannian manifold with bounded geometry.

A corollary is

Corollary 3.13. Let $M$ be a closed manifold and $F: M \times[0, T) \rightarrow N$ a smooth solution of the mean curvature flow on a maximal time interval in a complete Riemannian manifold $(N, g)$. If $\sup _{t \in[0, T)} \max _{M_{t}}|A|<\infty$, then $T=\infty$.

Note that long-time existence does not automatically imply convergence. For example, consider the surface of revolution $N \subset \mathbb{R}^{3}$ generated by the function $f(x)=1+e^{-x}$. A circle $\gamma$ of revolution moving by curve shortening flow on $N$ will then exist for all $t \in[0, \infty)$ with uniformly bounded curvature but it will not converge since it tends off to infinity. Some results on the regularity of curve shortening flow in high codimension have been derived in [CM07.

However, in some geometries once long-time existence is established one can use the Arzela-Ascoli theorem to extract convergent subsequences.

\subsection{Singularities.}

If a solution $F: M \times[0, T) \rightarrow N$ of the mean curvature flow exists only for finite time, then Proposition 3.11 implies the formation of a singularity. The question then arises how to understand the geometric and analytic nature of these singularities. From Proposition 3.11 we know that

$$
\limsup _{t \rightarrow T} \max _{M_{t}}|A|^{2}=\infty .
$$

One possible approach to classify singularities is to distinguish them by the blowup rate of $\max _{M_{t}}|A|^{2}$. The next definition originally appeared in Hui90 in the context of hypersurfaces in $\mathbb{R}^{m+1}$ but can be stated in the same way for arbitrary mean curvature flows.

Definition 3.14. Suppose $F: M \times[0, T) \rightarrow N$ is a smooth solution of the mean curvature flow with $T<\infty$ and

$$
\limsup _{t \rightarrow T} \max _{M_{t}}|A|^{2}=\infty
$$


a) A point $q \in N$ is called a blow-up point, if there exists a point $p \in M$ such that

$$
\lim _{t \rightarrow T} F(p, t)=q, \quad \lim _{t \rightarrow T}|A(p, t)|=\infty .
$$

b) One says that $M$ develops a singularity of Type I, if there exists a constant $c>0$ such that

$$
\max _{M_{t}}|A|^{2} \leq \frac{c}{T-t}, \forall t \in[0, T) .
$$

Otherwise one calls the singularity of Type II.

So if $q$ is a blow-up point then for $t \rightarrow T$ a singularity of Type I or Type II will form at $q \in N$ (and perhaps at other points as well).

In this context it is worth noting that the flow need not have a blow-up point in the sense of Definition 3.14, even if the second fundamental form blows up, e.g. the ambient space might have boundary or the singularity might form at spatial infinity. For this and other reasons it is appropriate to come up with more definitions. In Sto94, Stone introduced special and general singular points.

Definition 3.15. a) A point $p \in M$ is called a special singular point of the mean curvature flow, as $t \rightarrow T$, if there exists a sequence of times $t_{k} \rightarrow T$, such that

$$
\limsup _{k \rightarrow \infty}|A|\left(p, t_{k}\right)=\infty .
$$

b) A point $p \in M$ is called a general singular point of the mean curvature flow, as $t \rightarrow T$, if there exists a sequence of times $t_{k} \rightarrow T$ and a sequence of points $p_{k} \rightarrow p$, such that

$$
\limsup _{k \rightarrow \infty}|A|\left(p_{k}, t_{k}\right)=\infty .
$$

The reason to introduce the blow-up rate in Definition 3.14 is that for closed submanifolds in euclidean space one always has an analogue inequality in the other direction, i.e.

$$
\max _{M_{t}}|A|^{2} \geq \frac{\tilde{c}}{T-t}
$$

for some positive number $\tilde{c}$ (note that this does not necessarily hold, if the ambient space $N$ differs from $\mathbb{R}^{n}$ ). So in some sense singularities of Type I have the best controlled blow-up rate of $|A|^{2}$. Because of (30) one may actually refine the definition of special and general singular points for the mean curvature flow in $\mathbb{R}^{n}$, as was originally done by Stone in [Sto94. Instead of requiring $\lim \sup _{k \rightarrow \infty}|A|\left(p_{k}, t_{k}\right)=\infty$ one can define a general singular point $p \in M$ such that there exists some $\delta>0$ and a sequence $\left(p_{k}, t_{k}\right) \rightarrow(p, T)$ with

$$
|A|^{2}\left(p_{k}, t_{k}\right) \geq \frac{\delta}{T-t_{k}} .
$$

A sequence $\left(p_{k}, t_{k}\right)$ with this property is called an essential blow-up sequence. Although (30) gives a minimum blow-up rate for $\max _{p \in M}|A|^{2}(p, t)$ in the euclidean space, as $t$ approaches $T$, this does not rule out the possibility that, while $|A|^{2}(p, t) \geq \frac{\delta}{T-t}$ in some part of $M$, the blow-up of $|A|^{2}$ might simultaneously occur at some slower rate (say like $\left.(T-t)^{-\alpha}, \alpha \in(0,1)\right)$ somewhere else. Such "slowly forming singularities" would not be detected by a Type I blow-up procedure (see 
below) since the rescaling would be too fast. It is therefore interesting to understand, if this phenomenon occurs at all. As was recently shown by Le and Sesum LS10b this does not happen in the case of Type I singularities of hypersurfaces in $\mathbb{R}^{m+1}$ and all notions of singular sets defined in [Sto94] coincide. In particular they prove that the blow-up rate of the mean curvature must coincide with the blow-up rate of the second fundamental form, if a singularity of Type I is forming. We also mention that there exist many similarities between the formation of singularities in mean curvature flow and Ricci flow (see [EMT10] for a nice overview on Type I singularities in Ricci flow).

Type I: Let us now assume that $q \in \mathbb{R}^{n}$ is a blow-up point of Type I of $F$ : $M \times[0, T) \rightarrow \mathbb{R}^{n}$ and that $\operatorname{dim} M=m$. Huisken introduced the following rescaling technique in [Hui90] for hypersurfaces, but obviously it can be done in the same way for any codimension in $\mathbb{R}^{n}$ : Define an immersion $\tilde{F}: M \times[-1 / 2 \log T, \infty) \rightarrow \mathbb{R}^{n}$ by

$$
\tilde{F}(\cdot, s):=(2(T-t))^{-1 / 2}(F(\cdot, t)-q), \quad s(t)=-\frac{1}{2} \log (T-t) .
$$

One can then compute that $\tilde{F}$ satisfies the rescaled flow equation

$$
\frac{d}{d s} \tilde{F}=\tilde{\vec{H}}+\tilde{F}
$$

Since by assumption $|A|^{2} \leq c /(T-t)$ the second fundamental tensor $\tilde{A}$ of the rescaling is uniformly bounded in space and time. To study the geometric and analytic behavior of the rescaled immersions $\tilde{M}_{s}=\tilde{F}(M, s)$, Huisken proved a monotonicity formula for hypersurfaces in $\mathbb{R}^{n}$ moving by mean curvature. The corresponding result in arbitrary dimension and codimension is as follows: For $t_{0} \in \mathbb{R}$ let

$$
\rho: \mathbb{R}^{n} \times \mathbb{R} \backslash\left\{t_{0}\right\}:=\frac{1}{\left(4 \pi\left(t_{0}-t\right)\right)^{\frac{m}{2}}} e^{-\frac{|y|^{2}}{4\left(t_{0}-t\right)}} .
$$

Then $\rho_{\mid \mathbb{R}^{m} \times \mathbb{R} \backslash\left\{t_{0}\right\}}$ is the backward heat kernel of $\mathbb{R}^{m}$ at $\left(0, t_{0}\right)$ and the following monotonicity formula holds

Proposition 3.16 (Monotonicity formula (cf. Huisken Hui90])). Let $F: M \times$ $[0, T) \rightarrow \mathbb{R}^{n}$ be a smooth solution of the mean curvature flow and let $M$ be closed and $m$-dimensional. Then

$$
\frac{d}{d t} \int_{M} \rho(F(p, t), t) d \mu(p, t)=-\int_{M}\left|\vec{H}(p, t)+\frac{F^{\perp}(p, t)}{2\left(t_{0}-t\right)}\right|^{2} \rho(F(p, t), t) d \mu(p, t),
$$

where $d \mu(\cdot, t)$ denotes the volume element on $M$ induced by the immersion $F(\cdot, t)$ and $F^{\perp}$ denotes the normal part of the position vector $F$.

The proof is a simple consequence of

$$
\frac{d}{d t} \rho=\left(\frac{m}{2\left(t_{0}-t\right)}-\frac{|F|^{2}}{4\left(t_{0}-t\right)^{2}}-\frac{\langle F, \vec{H}\rangle}{2\left(t_{0}-t\right)}\right) \rho
$$

and

$$
\Delta \rho=\left(-\frac{m}{2\left(t_{0}-t\right)}+\frac{\left|F^{\top}\right|^{2}}{4\left(t_{0}-t\right)^{2}}-\frac{\langle F, \vec{H}\rangle}{2\left(t_{0}-t\right)}\right) \rho
$$


so that by the divergence theorem and from $\frac{d}{d t} d \mu=-|\vec{H}|^{2} d \mu$ we get

$$
\frac{d}{d t} \int_{M} \rho d \mu=\int_{M}\left(\frac{d}{d t} \rho+\Delta \rho-|\vec{H}|^{2} \rho\right) d \mu=-\int_{M}\left|\vec{H}+\frac{F^{\perp}}{2\left(t_{0}-t\right)}\right|^{2} \rho d \mu .
$$

Though the proof is easy, it is not obvious to look at the backward heat kernel when studying the mean curvature flow. This nice formula was used by Huisken to study the asymptotic behavior of the Type I blow-up and he proved the following beautiful theorem for hypersurfaces which again holds in arbitrary codimension

Proposition 3.17 (Type I blow-up (cf. Huisken Hui90])). Suppose $F: M \times$ $[0, T) \rightarrow \mathbb{R}^{n}$ is a smooth solution of the mean curvature flow of a closed $m$ dimensional smooth manifold $M$. Further assume that $T<\infty$ is finite and that $0 \in \mathbb{R}^{n}$ is a Type I blow-up point as $t \rightarrow T$. Then for any sequence $s_{j}$ there is a subsequence $s_{j_{k}}$ such that the rescaled immersed submanifolds $\tilde{M}_{s_{j_{k}}}$ converge smoothly to an immersed nonempty limiting submanifold $\tilde{M}_{\infty}$. Any such limit satisfies the equation

$$
\tilde{\vec{H}}+\tilde{F}^{\perp}=0 .
$$

Note that by Proposition 3.3 it is no restriction to assume that the blow-up point coincides with the origin. In general the limiting submanifold $\tilde{M}_{\infty}$ need not have the same topology as $M$, for example compactness might no longer hold. In addition it is unclear, if all solutions of (31) occur as blow-up limits of Type I singularities of compact submanifolds.

A solution of (31) is called a self-similar shrinking solution (or self-shrinker for short) of the mean curvature flow. Namely, one easily proves that a solution of (31) shrinks homothetically under the mean curvature flow and that there is a smooth positive function $c$ explicitly computable from the initial data and depending on the rescaled time $s$ such that

$$
\tilde{\vec{H}}_{s}+c(s) \tilde{F}_{s}^{\perp}=0 .
$$

There exists another interesting class of self-similar solutions of the mean curvature flow. These are characterized by the elliptic equation

$$
\vec{H}-F^{\perp}=0
$$

and are called self-expanders. In [EH89] Ecker and Huisken proved that entire graphs in $\mathbb{R}^{m+1}$ (in codimension 1 ) approach asymptotically expanding self-similar solutions if they satisfy a certain growth condition at infinity. Later Stavrou [Sta98] proved the same result under the weaker assumption that the graph has bounded gradient and a unique cone at infinity. Furthermore, he gave a characterization of expanding self-similar solutions to mean curvature flow with bounded gradient.

A classification of self-shrinking or self-expanding solutions is far from being complete. However there are some special situations for which one can say something. Self-shrinking curves have been completely classified by Abresch and Langer in AL86. Though their proof has been carried out for the curve shortening flow in $\mathbb{R}^{2}$ the result also applies to arbitrary codimension since (31) becomes an ODE for $m=1$ and the solutions are uniquely determined by their position and velocity 
vectors so that all 1-dimensional solutions of (31) must be planar. For hypersurfaces there exists a beautiful theorem by Huisken in Hui93 that describes all self-shrinking hypersurfaces with nonnegative (scalar) mean curvature. Later this result could be generalized by the author in the following sense

Proposition 3.18 ([Smo05]). For a closed immersion $M^{m} \subset \mathbb{R}^{n}, m \geq 2$ are equivalent:

a) $M$ is a self-shrinker of the mean curvature flow with nowhere vanishing mean curvature vector $\vec{H}$ and the principal normal vector $\nu:=\vec{H} /|\vec{H}|$ is parallel in the normal bundle.

b) $M$ is a minimal immersion in a round sphere.

In the same paper one finds a similar description for the non-compact case.

Type I singularities usually occur when there exists some kind of pinching of the second fundamental form and such situations occur quite often (cf. subsection 4). It is therefore surprising that there are situations, where one can exclude Type I singularities at all. In Smo00, Theorem 2.3.5] it was shown that there do not exist any compact Lagrangian solutions of (31) with trivial Maslov class $m_{1}=[H / \pi]=0$. Wang Wan01a and Chen \& Li CL04 observed that finite time Type I singularities of the Lagrangian mean curvature flow of closed Lagrangian submanifolds can be excluded, if the initial Lagrangian is almost calibrated in the sense that $* \operatorname{Re}\left(d z_{\mid M}\right)>0$. The condition to be almost calibrated is equivalent to the assumption that the Maslov class is trivial and that the Lagrangian angle $\alpha$ satisfies $\cos \alpha>0$. The difference of the results of Wang, Chen and Li in Wan01a,CL04 w.r.t. the result in Smo00] is, that the blow-up need not be compact any more. Later Neves Nev07] extended this result to the case of zero Maslov class, i.e. to the case where a globally defined Lagrangian angle $\alpha$ exists on $M$, thus removing the almost calibrated condition. In GSSZ07, Theorem 1.9] we proved a classification result for Lagrangian self-shrinkers and expanders in case they are entire graphs with a growth condition at infinity. In these cases Lagrangian self-similar solutions must be minimal Lagrangian cones.

Therefore when we study the Lagrangian mean curvature flow of closed Lagrangian submanifolds with trivial Maslov class we need to consider singularities of Type II only.

Type II: To study the shape of the submanifold near a singularity of Type II one can define a different family of rescaled flows. Following an idea of Hamilton Ham95a one can choose a sequence $\left(p_{k}, t_{k}\right)$ as follows: For any integer $k \geq 1$ let $t_{k} \in[0, T-1 / k], p_{k} \in M$ be such that

$$
\left|A\left(p_{k}, t_{k}\right)\right|^{2}\left(T-\frac{1}{k}-t_{k}\right)=\max _{\substack{t \leq T-1 / k \\ p \in M}}|A(p, t)|^{2}\left(T-\frac{1}{k}-t\right) .
$$

Furthermore one chooses

$$
L_{k}=\left|A\left(p_{k}, t_{k}\right)\right|, \quad \alpha_{k}=-L_{k}^{2} t_{k}, \quad \omega_{k}=L_{k}^{2}\left(T-t_{k}-1 / k\right) .
$$


If the singularity is of Type II then one has

$$
t_{k} \rightarrow T, \quad L_{k} \rightarrow \infty, \quad \alpha_{k} \rightarrow-\infty, \quad \omega_{k} \rightarrow \infty .
$$

Instead of $|A|$ one may use other quantities in the definition of these sequences, if it's known that they blow-up with a certain rate as $t \rightarrow T$. For example, in HS99a the mean curvature $H$ was used in the case of mean convex hypersurfaces in $\mathbb{R}^{m+1}$.

Then one can consider the following rescaling: For any $k \geq 1$, let $M_{k, \tau}$ be the family of submanifolds defined by the immersions

$$
F_{k}(\cdot, \tau):=L_{k}\left(F\left(\cdot, L_{k}^{-2} \tau+t_{k}\right)-F\left(p_{k}, t_{k}\right)\right), \quad \tau \in\left[\alpha_{k}, \omega_{k}\right] .
$$

The proper choice of the blow-up quantity $(|A|, H$ or similar $)$ in the definition of the rescaling will be essential to describe its behavior. Besides this rescaling technique there exist other methods to rescale singularities and the proper choice of the rescaling procedure depends on the particular situation in which the flow is considered. A nice reference for some of the scaling techniques is [Eck04.

If $M$ is compact and develops a Type II singularity then a subsequence of the flows $M_{k, \tau}$ converges smoothly to an eternal mean curvature flow $\tilde{M}_{\tau}$ defined for all $\tau \in \mathbb{R}$. Then a classification of Type II singularities depends on the classification of eternal solutions of the mean curvature flow.

In $\mathbb{R}^{2}$ the only convex eternal solution (up to scaling) of the mean curvature flow is given by the "grim reaper"

$$
y=-\log \cos x / \pi .
$$

The grim reaper is a translating soliton of the mean curvature flow, i.e. it satisfies the geometric PDE

$$
\vec{H}=V^{\perp},
$$

for some fixed vector $V \in \mathbb{R}^{n}$. A translating soliton moves with constant speed in direction of $V$.

In AV97 the authors constructed some particular solutions of the mean curvature flow that develop Type II singularities. In $\mathbb{R}^{2}$ examples of curves that develop a Type II singularity are given by some cardioids Ang91. Using a Harnack inequality, Hamilton Ham95b proved that any eternal convex solution of the mean curvature flow of hypersurfaces in $\mathbb{R}^{m+1}$ must be a translating soliton, if it assumes its maximal curvature at some point in space-time. In CJL05 the authors study whether such convex translating solutions are rotationally symmetric, and if every 2-dimensional rotationally symmetric translating soliton is strictly convex.

Various different notions of weak solution have been developed to extend the flow beyond the singular time $T$, including the geometric measure theoretic solutions of Brakke Bra78 and the level set solutions of Chen, Giga \& Goto CGG91 and Evans \& Spruck ES91, which were subsequently studied further by Ilmanen Ilm92. In HS09. Huisken and Sinestrari define such a notion based on a surgery procedure. 


\section{SPECIAL RESUlts IN HIGHER CODIMENSION}

In this chapter we mention the most important results in mean curvature flow that depend on more specific geometric situations and we will focus on results in higher codimension, especially on graphs and results in Lagrangian mean curvature flow.

\subsection{Preserved classes of immersions.}

Definition 4.1. Let $\mathscr{I}$ be the class of smooth $m$-dimensional immersions into a Riemannian manifold $(N, g)$ and suppose $\mathscr{F} \subset \mathscr{I}$ is a subclass. We say that $\mathscr{F}$ is a preserved class under the mean curvature flow, if for any solution $F_{t}: M \rightarrow N$, $t \in[0, T)$ of the mean curvature flow with $\left(F_{0}: M \rightarrow N\right) \in \mathscr{F}$ we also have $\left(F_{t}: M \rightarrow N\right) \in \mathscr{F}$ for all $t \in[0, T)$.

Preserved classes of the mean curvature flow are very important since one can often prove special results within these classes. Many classes can be expressed in terms of algebraic properties of the second fundamental form and in general it is a hard problem to detect those classes. We give a number of examples

\section{Example 4.2.}

a) $\mathscr{F}_{1}:=\left\{\right.$ Convex hypersurfaces in $\left.\mathbb{R}^{m+1}\right\}$

b) $\mathscr{F}_{2}:=\left\{\right.$ Mean convex hypersurfaces in $\mathbb{R}^{m+1}$, i.e. $\left.H>0\right\}$

c) $\mathscr{F}_{3}:=\{$ Embedded hypersurfaces in Riemannian manifolds

d) $\mathscr{F}_{4}:=\left\{\right.$ Hypersurfaces in $\mathbb{R}^{m+1}$ as entire graphs over a flat plane $\}$

e) $\mathscr{F}_{5}:=\{$ Lagrangian immersions in Kähler-Einstein manifolds $\}$

To prove that classes are preserved one often uses the parabolic maximum principle (at least in the compact case). Besides the classical maximum principle for scalar quantities there exists an important maximum principle for bilinear forms due to Richard Hamilton that was originally proven in Ham82b] and improved in Ham86.

Another very important property is the pinching property of certain classes of immersions in $\mathbb{R}^{n}$.

Definition 4.3. Let $F: M \rightarrow \mathbb{R}^{n}$ be a (smooth) immersion. We say that the second fundamental form $A$ of $F$ is $\delta$-pinched, if the inequality

$$
|A|^{2} \leq \delta|\vec{H}|^{2}
$$

holds everywhere on $M$.

From

$$
0 \leq\left|A-\frac{1}{m} \vec{H} \otimes F^{*} g\right|^{2}=|A|^{2}-\frac{1}{m}|\vec{H}|^{2}
$$

with $m=\operatorname{dim} M$ we immediately obtain that $\delta$ is bounded from below by $1 / m$.

For hypersurfaces in $\mathbb{R}^{m+1}$ it is known:

Proposition 4.4. Let $\delta \geq 1 / m$. The class of closed $\delta$-pinched hypersurfaces in $\mathbb{R}^{m+1}$ is a preserved class under the mean curvature flow. 
Proof. This easily follows from the maximum principle and the evolution equation for $f:=|A|^{2} / H^{2}$.

It can be shown that an $m$-dimensional submanifold in $\mathbb{R}^{n}$ is $1 / m$-pinched, if and only if it is either a part of a round sphere or a flat subspace. Therefore closed pinched submanifolds are in some sense close to spheres. In some cases this pinching can improve under the mean curvature flow. To explain this in more detail, we make the following definition: Let $\mathscr{F}$ be a nonempty class of smooth $m$-dimensional immersions $F: M \rightarrow \mathbb{R}^{n}$, where $M$ is not necessarily fixed, and set

$$
\delta_{\mathscr{F}}:=\sup \left\{\delta \in \mathbb{R}:\left|A_{F}(p)\right|^{2} \geq \delta\left|\vec{H}_{F}(p)\right|^{2}, \forall p \in M, \forall\left(F: M \rightarrow \mathbb{R}^{n}\right) \in \mathscr{F}\right\},
$$

where $A_{F}$ and $\vec{H}_{F}$ denote the second fundamental form and mean curvature vector of the immersion $F: M \rightarrow \mathbb{R}^{n}$. Then $\delta_{\mathscr{F}} \geq \frac{1}{m}$ and $\delta_{\mathscr{F}}$ is finite, if and only if $\mathscr{F}$ contains an immersion $F: M \rightarrow \mathbb{R}^{n}$ for which $\vec{H}_{F}$ does not vanish completely.

Definition 4.5. Let $\mathscr{F}$ be a preserved class of smooth $m$-dimensional immersions with $\delta_{\mathscr{F}}<\infty$ and suppose $\delta$ is some real number with $\delta>\delta_{\mathscr{F}}$. We say that $\mathscr{F}$ is $\delta$-pinchable, if for any $\epsilon$ with $0 \leq \epsilon<\delta-\delta_{\mathscr{F}}$ the class

$$
\mathscr{F}_{\epsilon}:=\left\{\left(F: M \rightarrow \mathbb{R}^{n}\right) \in \mathscr{F}:\left|A_{F}(p)\right|^{2} \leq\left(\delta_{\mathscr{F}}+\epsilon\right)\left|\vec{H}_{F}(p)\right|^{2}, \forall p \in M\right\}
$$

is a preserved class under the mean curvature flow.

Example 4.6. a) It follows from Theorem 4.4 that the class $\mathscr{F}(m, m+1)$ of smooth $m$-dimensional closed immersions into $\mathbb{R}^{m+1}$ is $\delta$-pinchable for any $\delta \geq$ $1 / m=\delta_{\mathscr{F}(m, m+1)}$ and that the pinching constant $\delta_{\mathscr{F}(m, m+1)}$ is attained if and only if the immersion $F: M \rightarrow \mathbb{R}^{n}$ is a round sphere or a flat plane (or part of).

b) A beautiful result recently obtained by Andrews and Baker [AB10] shows that the class $\mathscr{F}(m, m+k)$ of smooth $m$-dimensional closed immersions into $\mathbb{R}^{m+k}$ is $\delta$-pinchable with $\delta=1 /(m-1)$, if $m \geq 4$ and with $\delta=4 / 3 m$ for $2 \leq m \leq 4$. Here $\delta_{\mathscr{F}(m, m+k)}=1 / m$. They prove that $\delta$-pinched immersions contract to round points. Thus for such immersions one has $M=S^{m}$ and they are smoothly homotopic to hyperspheres.

We will now show that the class $\mathscr{L}(m)$ of smooth closed Lagrangian immersions into $\mathbb{C}^{m}$ is not $\delta$-pinchable for any $\delta$.

Theorem 4.7. Let $\mathscr{L}(m)$ be the class of smooth closed Lagrangian immersions into $\mathbb{C}^{m}, m>1$. Then $\delta_{\mathscr{L}(m)}=3 /(m+2)$ and $\mathscr{L}(m)$ is not $\delta$-pinchable for any $\delta$.

Proof. Given a Lagrangian immersion $F: M \rightarrow \mathbb{C}^{m}$ we have

$$
0 \leq\left|h_{i j k}-\frac{1}{m+2}\left(H_{i} g_{j k}+H_{j} g_{k i}+H_{k} g_{i j}\right)\right|^{2}=|A|^{2}-\frac{3}{m+2}|\vec{H}|^{2},
$$

where $H_{i} d x^{i}$ is the mean curvature form. This implies $\delta_{\mathscr{L}(m)} \geq \frac{3}{m+2}$. On the other hand equality is attained for flat Lagrangian planes and for the Whitney spheres. These are given by restricting the immersions

$$
\tilde{F}_{r}: \mathbb{R}^{m+1} \rightarrow \mathbb{C}^{m}, \quad \tilde{F}_{r}\left(x^{1}, \ldots, x^{m+1}\right):=\frac{r\left(1+i x^{m+1}\right)}{1+\left(x^{m+1}\right)^{2}}\left(x^{1}, \ldots, x^{m}\right), \quad r>0
$$


to $S^{m} \subset \mathbb{R}^{m+1}$, i.e. $F_{r}:=\tilde{F}_{r \mid S^{m}}: S^{m} \rightarrow \mathbb{C}^{m}$ is a Lagrangian immersion of the sphere with $|A|^{2}=\frac{3}{m+2}|\vec{H}|^{2}$. The number $r$ is called the radius of the Whitney sphere. This shows $\delta_{\mathscr{L}(M)}=\frac{3}{m+2}$. It has been shown by Ros and Urbano in RU98 that Whitney spheres and flat Lagrangian planes are the only Lagrangian submanifolds in $\mathbb{C}^{m}, m>1$, for which $|A|^{2}=\frac{3}{m+2}|\vec{H}|^{2}$. Now if $\mathscr{L}(M)$ would be $\delta$ pinchable for some $\delta$, then in particular the Lagrangian mean curvature flow would preserve the identity $|A|^{2}=\frac{3}{m+2}|\vec{H}|^{2}$. This is certainly true for the flat planes but for the Whitney sphere this cannot be true. Because the result of Ros and Urbano implies that under the assumption of $\delta$-pinchability a Whitney sphere would then stay a Whitney sphere under the Lagrangian mean curvature flow and the radius of the spheres would decrease. In other words, the Whitney sphere would have to be a self-similar shrinking solution of the Lagrangian mean curvature flow. This is a contradiction to the well-known result (first shown in Smo00, Corollary 2.3.6]), that there are no self-shrinking Lagrangian spheres in $\mathbb{C}^{m}$, if $m>1$.

\subsection{Lagrangian mean curvature flow.}

In this subsection we will assume that $F: M \rightarrow N$ is a closed smooth Lagrangian immersion into a Kähler manifold $(N, g, J)$. It has been shown in Smo96] that the Lagrangian condition is preserved, if the ambient Kähler manifold is Einstein. This includes the important case of Calabi-Yau manifolds, i.e. of Ricci flat Kähler manifolds. Recently a generalized Lagrangian mean curvature flow in almost Kähler manifolds with Einstein connections has been defined by Wang and the author in SW11. This generalizes an earlier result by Behrndt Beh08. The Einstein condition is relevant in view of the Codazzi equation which implies that the mean curvature form is closed, a necessary condition to guarantee that the deformation is Lagrangian. To explain this in more detail, observe that the symplectic form $\omega$ induces an isomorphism between the space of smooth normal vector fields along $M$, and the space of smooth 1-forms on $M$. Namely, given $\theta \in \Omega^{1}(M)$ there exists a unique normal vector field $V \in \Gamma\left(T^{\perp} M\right)$ with $\theta=\omega(\cdot, V)$. If $F: M \times[0, T) \rightarrow N$ is a smooth family of Lagrangian immersions evolving in normal direction driven by some smooth time depending 1 -forms $\theta \in \Omega^{1}(M)$ we have

$$
0=\frac{d}{d t} F^{*} \omega=d\left(\omega\left(\frac{d}{d t} F, \cdot\right)\right)=-d \theta
$$

and consequently $\theta$ must be closed. Since the mean curvature form is given by

$$
H=\omega(\cdot, \vec{H})
$$

we obtain that the closeness of $H$ is necessary to guarantee that the mean curvature flow preserves the Lagrangian condition, and it is indeed sufficient ([Smo96, Smo00]). In the non-compact case this is open in general, but in some cases (like graphs over complete Lagrangian submanifolds with bounded geometry) this can be reduced to the existence problem of solutions to a parabolic equation of Monge-Ampère type. The Lagrangian condition can be interpreted as an integrability condition. For example, if $M$ is a graph in $\mathbb{C}^{m}=\mathbb{R}^{m} \oplus i \mathbb{R}^{m}$ over the real part, i.e. if $M$ is the image of some embedding

$$
F: \mathbb{R}^{m} \rightarrow \mathbb{C}^{m}, \quad F(x)=x+i y(x),
$$


where $y=y_{i} d x^{i}$ is a smooth 1 -form on $\mathbb{R}^{m}$, then $M$ is Lagrangian if and only if $y$ is closed. Consequently there exists a smooth function $u$ (called a generating function) such that $y=d u$. Assuming that $M$ evolves under the mean curvature flow and that all subsequent graphs $M_{t}$ are still Lagrangian one can integrate the evolution equation of $y=d u$ and obtains a parabolic evolution equation of MongeAmpère type for $u$. Conversely, given a solution $u$ of this parabolic Monge-Ampère type equation on $\mathbb{R}^{m}$ one can generate Lagrangian graphs $F=(x, d u)$ and it can be shown that these graphs move under the mean curvature flow (cf. [Smo00]). The same principle works in a much more general context, namely if the initial Lagrangian submanifold lies in some Kähler-Einstein manifold and the Lagrangian has bounded geometry. The boundedness of the geometry is essential for the proof since this allows to exploit the implicit function theorem to obtain the existence of a Monge-Ampère type equation similar as above.

This integrability property has one important consequence. In general, given a second order parabolic equation, one would need uniform $C^{2, \alpha}$-bounds of the solution in space and uniform $C^{1, \alpha}$-estimates in time to ensure long-time existence, as follows from Schauder theory. For the mean curvature flow these estimates are already induced by a uniform estimate of the second fundamental form $A$ (see Corollary 3.131$)$, so essentially by $C^{2}$-estimates. In the Lagrangian mean curvature flow $F: M \times[0, T) \rightarrow N$ one may instead use the parabolic equation of Monge-Ampère type for the generating function $u$ and consequently one just needs $C^{1, \alpha}$-estimates in space and $C^{0, \alpha}$ estimates in time for $F$ which itself is of first order in $u$. In some situations this principle has been used successfully, for example in SW02, Smo04. There it was shown that Lagrangian tori $M=T^{m}$ in flat tori $N=T^{2 m}$ converge to flat Lagrangian tori, if the universal cover possesses a convex generating function $u$. We also mention a recent generalization to the complete case by Chau, Chen and $\mathrm{He} \mathrm{CCH} 09 \mathrm{~b}$.

The evolution equations for the Lagrangian mean curvature flow have been derived in Smo00] (see also Smo96]) and can also be obtained directly from our general evolution equations stated in subsection 3.2 Besides the evolution equation for the induced metric the equation for the mean curvature form $H=H_{i} d x^{i}$ is perhaps the most important and is given by

$$
\nabla_{\frac{d}{d t}} H=d d^{\dagger} H+\frac{S}{2 m} H
$$

where $S$ denotes the scalar curvature of the ambient Kähler-Einstein manifold, $m$ is the dimension of the Lagrangian immersion and $d^{\dagger} H=\nabla^{i} H_{i}$. In particular it follows that the cohomology class $\left[H e^{-\frac{S}{2 m} t}\right]$ is invariant under the Lagrangian mean curvature flow and in a Calabi-Yau manifold the Lagrangian immersions with trivial first Maslov class $m_{1}$ (we have $m_{1}=\frac{1}{\pi}[H]$ ) form a preserved class. This also shows that if the scalar curvature $S$ is nonnegative, then a necessary condition to have long-time existence and smooth convergence of the Lagrangian mean curvature flow to a minimal Lagrangian immersion is that the initial mean curvature form is exact. Exactness of the mean curvature form will then be preserved and a globally defined Lagrangian angle $\alpha$ with $d \alpha=H$ exists for all $t$. This last result also holds for general scalar curvature $S$ and after choosing a proper gauge for $\alpha$ one can prove 
[Smo99, Lemma 2.4] that $\alpha$ satisfies the evolution equation

$$
\frac{d}{d t} \alpha=\Delta \alpha+\frac{S}{2 m} \alpha .
$$

It is then a simple consequence of the maximum principle that on compact Lagrangian submanifolds $M$ with trivial Maslov class in a Calabi-Yau manifold there exist uniform upper and lower bounds for the Lagrangian angle given by its initial maximum resp. minimum. In particular, the condition to be almost calibrated, i.e. $* \operatorname{Re}\left(d z_{\mid M}\right)=\cos \alpha>0$ is preserved. Here $d z$ denotes the complex volume form on the Calabi-Yau manifold and it is well known that the Lagrangian angle $\alpha$ satisfies

$$
d z_{\mid M}=e^{i \alpha} d \mu,
$$

where $d \mu$ is the volume form on $M$. Almost calibrated Lagrangian submanifolds in Calabi-Yau manifolds have some nice properties under the mean curvature flow. As was mentioned earlier, from the results in CL04, Nev07, Smo00, Wan01a we know that singularities of the Lagrangian mean curvature flow of compact Lagrangian immersions with trivial Maslov class in Calabi-Yau manifolds cannot be of Type I and therefore a big class of singularities is excluded. So far one cannot say much about singularities of Type II and in particular, one does not know if they occur at all in the case of compact almost calibrated Lagrangians (though some authors have some rather heuristic arguments for the existence of such singularities). It is worth noting that there do not exist any compact almost calibrated Lagrangian immersions in $\mathbb{R}^{2 m}$ (but in $\mathbb{T}^{2 m}$ they exist). In [Smo02, Theorem 1.3] it was shown that there exists a uniform (in time) lower bound for the volume of a compact almost calibrated Lagrangian evolving by its mean curvature in a Calabi-Yau (and more generally in a Kähler-Einstein manifold of non-positive scalar curvature).

An interesting class of Lagrangian immersions is given by monotone Lagrangians. A Lagrangian immersion $F: M \rightarrow \mathbb{R}^{2 m}$ is called monotone, if

$$
[H]=\epsilon\left[F^{*} \lambda\right],
$$

for some positive constant $\epsilon$ (called monotonicity constant). Here $\lambda$ is the Liouville form on $\mathbb{R}^{2 m}=T \mathbb{R}^{m}$. In GSSZ07 we proved several theorems concerning monotone Lagrangian immersions. From the evolution equations of $H$ and $F^{*} \lambda$ one derives that monotonicity is preserved with a time dependent monotonicity constant $\epsilon(t)$. Gromov Gro85 proved that given an embedded Lagrangian submanifold $M$ in $\mathbb{R}^{2 m}$ there exists a holomorphic disc with boundary on $M$. On the other hand, from the evolution equations of $H$ and $F^{*} \lambda$ we get that the area of holomorphic discs with boundary representing some fixed homology class in $M$ is shrinking linearly in time. If the Lagrangian is monotone, then the shrinking rate for the area of holomorphic discs is the same for all homology classes.

Unfortunately it is unknown, if embeddedness of Lagrangian submanifolds is preserved under mean curvature flow (in general, embeddedness in higher codimension is not preserved but self-intersection numbers might be). Suppose $F: M \times[0, T) \rightarrow$ $\mathbb{R}^{2 m}$ is a Lagrangian mean curvature flow of a compact monotone Lagrangian with initial monotonicity constant $\epsilon>0$ and suppose $0<T_{e} \leq T$ is the embedding time, i.e. the maximal time such that $F_{t}: M \rightarrow \mathbb{R}^{2 m}$ is an embedding for all $0 \leq t<T_{e}$. 
Then we proved [GSSZ07, Theorem 1.6 and Theorem 1.11] that $T_{e} \leq \frac{1}{\epsilon}$. Moreover

$$
T=\frac{1}{\epsilon},
$$

in case $T_{e}=T$ and if $M$ develops a Type I singularity as $t \rightarrow T$. We note that this result is rather unique in mean curvature flow. Usually it is not possible to explicitly determine the span of life of a solution and to determine it in terms of its initial data. In the same paper we also proved the existence of compact embedded monotone Lagrangian submanifolds (even with some additional symmetry) that develop Type II singularities and consequently it is not true that monotone embedded Lagrangian submanifolds must develop Type I singularities, as was conjectured earlier by some people.

Lagrangian submanifolds appear naturally in another context. If

$$
f: M \rightarrow K
$$

is a symplectomorphism between two symplectic manifolds $\left(M, \omega^{M}\right),\left(K, \omega^{K}\right)$ then the graph

$$
F: M \rightarrow M \times K, \quad F(p)=(p, f(p))
$$

is a Lagrangian embedding in $\left(M \times K,\left(\omega^{M},-\omega^{K}\right)\right)$.

If $\left(M, \omega^{M}, J^{M}, g^{M}\right)$ and $\left(K, \omega^{K}, J^{K}, g^{K}\right)$ are both Kähler-Einstein, then the product manifold is Kähler-Einstein as well and one can use the Lagrangian mean curvature flow to deform a symplectomorphism. In Smo02 symplectomorphisms between Riemann surfaces of the same constant curvature $S$ have been studied and it was shown (Lemma 10 and Lemma 14) that Lagrangian graphs that come from symplectomorphisms stay graphs for all time. The same result was obtained independently by Wang in [Wan01b] (the quantities $r$ in [Smo02, Lemma 10] and $\eta$ in [Wan01b, Proposition 2.1] are the same up to some positive constant). In Smo02 the graphical condition was then used in the case of non-positive curvature $S$ and under the angle condition $\cos \alpha>0$ (almost calibrated) to derive explicit bounds for the second fundamental form and to establish long-time existence and smooth convergence to a minimal Lagrangian surface. Wang used the graphical condition in Wan01b to obtain long-time existence without a sign condition on $S$ by methods related to White's regularity theorem and then proved convergence of subsequences to minimal Lagrangian surfaces. Later he refined his result and proved smooth convergence in Wan08a. In a recent paper by Medos and Wang MW09 it is shown that symplectomorphisms of $\mathbb{C P}^{m}$ for which the singular values satisfy some pinching condition can be smoothly deformed into a biholomorphic isometry of $\mathbb{C P}^{m}$.

In a joint paper [SW02] (see also Smo04]) Wang and the author studied Lagrangian graphs in the cotangent bundle of a flat torus and proved that Lagrangian tori with a convex generating function converge smoothly to a flat Lagrangian torus. In this case the convexity of the generating function $u$ implies that the Monge-Ampère type operator that appears in the evolution equation of $u$ becomes concave and then results of Krylov Kry87 imply uniform $C^{2, \alpha}$-estimates in space and $C^{1, \alpha_{-}}$ estimates in time and long-time existence and convergence follows. A similar result holds for non-compact graphs CCH09b. 


\subsection{Mean curvature flow of graphs.}

As the results mentioned at the end of the last subsection show, mean curvature flow of graphs behaves much "nicer" than in the general case. There are many results for graphs moving under mean curvature flow. The first result in this direction was the paper by Ecker and Huisken [EH89] where long-time existence of entire graphs in $\mathbb{R}^{m+1}$ (hypersurfaces) was shown. Convergence to flat subspaces follows, if the growth rate at infinity is linear. Under a different growth rate they prove that the hypersurfaces converge asymptotically to entire self-expanding solutions of the mean curvature flow. The crucial observation in their paper was that the angle function $v:=\langle\nu, Z\rangle$ (scalar product of the unit normal and the height vector $Z$ ) satisfies a very useful evolution equation that can be exploited to bound the second fundamental form appropriately.

Many results in mean curvature flow of graphs have been obtained by Wang. For example in Wan02 he studied the graph induced by a map $f: M \rightarrow K$ between to Riemannian manifolds of constant sectional curvatures. Under suitable assumptions on the differential of $f$ and the curvatures of $M$ resp. $K$ he obtained long-time existence and convergence to constant maps. In [TW04 the authors consider a graph in the product $M \times K$ of two Riemannian manifolds of constant sectional curvatures. A map $f: M \rightarrow K$ for which the singular values $\lambda_{i}$ of $f$ satisfy the condition $\lambda_{i} \lambda_{j}<1$ for all $i \neq j$ is called an area decreasing map. The main theorem in their paper states long-time existence of the mean curvature flow and convergence to a constant map under the following assumptions:

i) the initial graph of $f$ is area-decreasing;

ii) $\sigma^{M} \geq\left|\sigma^{K}\right|, \sigma^{M}+\sigma^{K}>0$ and $\operatorname{dim} M \geq 2$,

where $\sigma^{M}, \sigma^{K}$ denote the sectional curvatures of $M$ resp. $K$. In particular area decreasing maps from $S^{m}$ to $S^{k}$ are homotopically trivial for $m \geq 2$.

In LL03 graphs in Riemannian products of two space forms have been studied and under certain assumptions on the initial graph long-time existence was established. In Wan05] two long-time existence and convergence results for the mean curvature flow of graphs induced by maps $f: M \rightarrow K$ between two compact Riemannian manifolds of dimension $m=\operatorname{dim} M \geq 2$ and $\operatorname{dim} K=2$ are given. In the first theorem $M$ and $K$ are assumed to be flat, and in the second theorem, $M=S^{m}$ is an $m$-sphere of constant curvature $k_{1}>0$ and $K$ a compact surface of constant curvature $k_{2}$ with $\left|k_{2}\right| \leq k_{1}$. The key assumption on the graph is expressed in terms of the Gauß map, i.e. the map which assigns to a point $p$ its tangent space. The latter is an element of the bundle of $m$-dimensional subspaces of $T N, N=M \times K$ and it is shown that there exists a sub-bundle $\mathfrak{G}$ of $T N$ which is preserved along the mean curvature flow. The same author proved a beautiful general theorem for the Gauß map under the mean curvature flow (see Wan03]).

\section{REFERENCES}

[AL86] U. Abresch and J. Langer, The normalized curve shortening flow and homothetic solutions, J. Differential Geom. 23 (1986), no. 2, 175-196. 
[Alt91] St. J. Altschuler, Singularities of the curve shrinking flow for space curves, J. Differential Geom. 34 (1991), no. 2, 491-514.

[AG92] St. J. Altschuler and M. A. Grayson, Shortening space curves and flow through singularities, J. Differential Geom. 35 (1992), no. 2, 283-298.

[AS97] L. Ambrosio and H. M. Soner, A measure-theoretic approach to higher codimension mean curvature flows, Ann. Scuola Norm. Sup. Pisa Cl. Sci. (4) 25 (1997), no. 1-2, 27-49 (1998). Dedicated to Ennio De Giorgi.

[AB10] B. Andrews and C. Baker, Mean curvature flow of pinched submanifolds to spheres, J. Differential Geom. 85 (2010), no. 3, 357-395.

[Anc06] H. Anciaux, Construction of Lagrangian self-similar solutions to the mean curvature flow in $\mathbb{C}^{n}$, Geom. Dedicata 120 (2006), 37-48.

[Ang91] S. Angenent, On the formation of singularities in the curve shortening flow, J. Differential Geom. 33 (1991), no. 3, 601-633.

[AV97] S. B. Angenent and J. J. L. Velázquez, Degenerate neckpinches in mean curvature flow, J. Reine Angew. Math. 482 (1997), 15-66.

[Beh08] T. Behrndt, Generalized Lagrangian mean curvature flow in Kähler manifolds that are almost Einstein, arXiv:0812.4256, to appear in Proceedings of CDG 2009, Leibniz Universität Hannover (2008).

[Bra78] K. A. Brakke, The motion of a surface by its mean curvature, Mathematical Notes, vol. 20, Princeton University Press, Princeton, N.J., 1978.

[CL10] I. Castro and A. M. Lerma, Hamiltonian stationary self-similar solutions for Lagrangian mean curvature flow in the complex Euclidean plane, Proc. Amer. Math. Soc. 138 (2010), no. 5, 1821-1832.

[CCH09a] A. Chau, J. Chen, and W. He, Entire self-similar solutions to Lagrangian Mean curvature flow, arXiv:0905.3869 (2009).

[CCH09b] _ Lagrangian Mean Curvature flow for entire Lipschitz graphs, arXiv:0902.3300 (2009).

[CGG91] Y. Chen, Y. Giga, and S. Goto, Uniqueness and existence of viscosity solutions of generalized mean curvature flow equations, J. Differential Geom. 33 (1991), no. 3, 749-786.

[CJL05] X. Chen, H. Jian, and Q. Liu, Convexity and symmetry of translating solitons in mean curvature flows, Chinese Ann. Math. Ser. B 26 (2005), no. 3, 413-422.

[CL01] J. Chen and J. Li, Mean curvature flow of surface in 4-manifolds, Adv. Math. 163 (2001), no. 2, 287-309.

[CL04] Singularity of mean curvature flow of Lagrangian submanifolds, Invent. Math. 156 (2004), no. 1, 25-51.

[CLT02] J. Chen, J. Li, and G. Tian, Two-dimensional graphs moving by mean curvature flow, Acta Math. Sin. (Engl. Ser.) 18 (2002), no. 2, 209-224.

[CM07] D. Chen and L. Ma, Curve shortening in a Riemannian manifold, Ann. Mat. Pura Appl. (4) 186 (2007), no. 4, 663-684.

[CP09] J. Chen and C. Pang, Uniqueness of unbounded solutions of the Lagrangian mean curvature flow equation for graphs, C. R. Math. Acad. Sci. Paris 347 (2009), no. 1718, 1031-1034 (English, with English and French summaries).

[CY07] B.-L. Chen and L. Yin, Uniqueness and pseudolocality theorems of the mean curvature flow, Comm. Anal. Geom. 15 (2007), no. 3, 435-490.

[CZ01] K.-S. Chou and X.-P. Zhu, The curve shortening problem, Chapman \& Hall/CRC, Boca Raton, FL, 2001.

[CSS07] J. Clutterbuck, O.C. Schnürer, and F. Schulze, Stability of translating solutions to mean curvature flow, Calc. Var. Partial Differential Equations 29 (2007), no. 3, 281293.

[CM04] T. H. Colding and W. P. Minicozzi II, Sharp estimates for mean curvature flow of graphs, J. Reine Angew. Math. 574 (2004), 187-195.

[CM09] Generic mean curvature flow I; generic singularities, arXiv:0908.3788 (2009).

[Eck82] K. Ecker, Estimates for evolutionary surfaces of prescribed mean curvature, Math. Z. 180 (1982), no. 2, 179-192.

[Eck01] A local monotonicity formula for mean curvature flow, Ann. of Math. (2) 154 (2001), no. 2, 503-525. 
[Eck04] Regularity theory for mean curvature flow, Progress in Nonlinear Differential Equations and their Applications, 57, Birkhäuser Boston Inc., Boston, MA, 2004.

[EH89] K. Ecker and G. Huisken, Mean curvature evolution of entire graphs, Ann. of Math. (2) 130 (1989), no. 3, 453-471.

[EH91] Interior estimates for hypersurfaces moving by mean curvature, Invent. Math. 105 (1991), no. 3, 547-569.

[EKNT08] K. Ecker, D. Knopf, L. Ni, and P. Topping, Local monotonicity and mean value formulas for evolving Riemannian manifolds, J. Reine Angew. Math. 616 (2008), 89-130.

[EMT10] J. Enders, R. Müller, and P. Topping, On Type I Singularities in Ricci flow, arXiv:1005.1624 (2010).

[ES91] L. C. Evans and J. Spruck, Motion of level sets by mean curvature. I, J. Differential Geom. 33 (1991), no. 3, 635-681.

[Gag84] M. E. Gage, Curve shortening makes convex curves circular, Invent. Math. 76 (1984), no. 2, 357-364.

[GH86] M. Gage and R. S. Hamilton, The heat equation shrinking convex plane curves, J. Differential Geom. 23 (1986), no. 1, 69-96.

[Ger80] C. Gerhardt, Evolutionary surfaces of prescribed mean curvature, J. Differential Equations 36 (1980), no. 1, 139-172.

[Gra87] M. A. Grayson, The heat equation shrinks embedded plane curves to round points, J. Differential Geom. 26 (1987), no. 2, 285-314.

[Gra89] Shortening embedded curves, Ann. of Math. (2) 129 (1989), no. 1, 71-111.

[GSSZ07] K. Groh, M. Schwarz, K. Smoczyk, and K. Zehmisch, Mean curvature flow of monotone Lagrangian submanifolds, Math. Z. 257 (2007), no. 2, 295-327.

[Gro85] M. Gromov, Pseudoholomorphic curves in symplectic manifolds, Invent. Math. 82 (1985), no. 2, 307-347.

[Ham82a] R. S. Hamilton, The inverse function theorem of Nash and Moser, Bull. Amer. Math. Soc. (N.S.) 7 (1982), no. 1, 65-222.

[Ham82b] _ Three-manifolds with positive Ricci curvature, J. Differential Geom. 17 (1982), no. $2,255-306$

[Ham86] - Four-manifolds with positive curvature operator, J. Differential Geom. 24 (1986), no. 2, 153-179.

[Ham93] Monotonicity formulas for parabolic flows on manifolds, Comm. Anal. Geom. 1 (1993), no. 1, 127-137.

[Ham95a] _ The formation of singularities in the Ricci flow, Surveys in differential geometry, Vol. II (Cambridge, MA, 1993), Int. Press, Cambridge, MA, 1995, pp. 7-136.

[Ham95b] _ Harnack estimate for the mean curvature flow, J. Differential Geom. 41 (1995), no. 1, 215-226.

[HL09] X. Han and J. Li, Translating solitons to symplectic and Lagrangian mean curvature flows, Internat. J. Math. 20 (2009), no. 4, 443-458.

[Hui84] G. Huisken, Flow by mean curvature of convex surfaces into spheres, J. Differential Geom. 20 (1984), no. 1, 237-266.

[Hui86] _ Contracting convex hypersurfaces in Riemannian manifolds by their mean curvature, Invent. Math. 84 (1986), no. 3, 463-480.

[Hui90] - Asymptotic behavior for singularities of the mean curvature flow, J. Differential Geom. 31 (1990), no. 1, 285-299.

[Hui93] _ Local and global behaviour of hypersurfaces moving by mean curvature, Differential geometry: partial differential equations on manifolds (Los Angeles, CA, 1990), Proc. Sympos. Pure Math., vol. 54, Amer. Math. Soc., Providence, RI, 1993, pp. 175191.

[HS99a] G. Huisken and C. Sinestrari, Mean curvature flow singularities for mean convex surfaces, Calc. Var. Partial Differential Equations 8 (1999), no. 1, 1-14.

[HS99b] Convexity estimates for mean curvature flow and singularities of mean convex surfaces, Acta Math. 183 (1999), no. 1, 45-70.

[HS09] _ Mean curvature flow with surgeries of two-convex hypersurfaces, Invent. Math. 175 (2009), no. 1, 137-221.

[Ilm92] T. Ilmanen, Generalized flow of sets by mean curvature on a manifold, Indiana Univ. Math. J. 41 (1992), no. 3, 671-705. 
[JLT10] D. Joyce, Y.-I. Lee, and M.-P. Tsui, Self-similar solutions and translating solitons for Lagrangian mean curvature flow, J. Differential Geom. 84 (2010), no. 1, 127-161.

[Kry87] N. V. Krylov, Nonlinear elliptic and parabolic equations of the second order, Mathematics and its Applications (Soviet Series), vol. 7, D. Reidel Publishing Co., Dordrecht, 1987.

[LS10a] N. Q. Le and N. Sesum, The mean curvature at the first singular time of the mean curvature flow, Ann. Inst. H. Poincaré Anal. Non Linéaire 27 (2010), no. 6, 1441-1459.

[LS10b] Blow-up rate of the mean curvature during the mean curvature flow and a gap theorem for self-shrinkers, arXiv:1011.5245v1 (2010).

[LL03] J. Li and Y. Li, Mean curvature flow of graphs in $\Sigma_{1} \times \Sigma_{2}$, J. Partial Differential Equations 16 (2003), no. 3, 255-265.

[LXYZ11] K. Liu, H. Xu, F. Ye, and E. Zhao, The extension and convergence of mean curvature flow in higher codimension, arXiv:1104.0971v1 (2011).

[MW09] I. Medos and M.-T. Wang, Deforming symplectomorphisms of complex projective spaces by the mean curvature flow, Preprint (2009).

[Mul56] W. W. Mullins, Two-dimensional motion of idealized grain boundaries, J. Appl. Phys. 27 (1956), 900-904.

[Nev07] A. Neves, Singularities of Lagrangian mean curvature flow: zero-Maslov class case, Invent. Math. 168 (2007), no. 3, 449-484.

[Nev10] Singularities of Lagrangian mean curvature flow: monotone case, Math. Res. Lett. 17 (2010), no. 1, 109-126.

[Per02] G. Perelman, The entropy formula for the Ricci flow and its geometric applications, arXiv:math/0211159 (2002).

[Per03a] — Ricci flow with surgery on three-manifolds, arXiv:math/0303109 (2003).

[Per03b] Finite extinction time for the solutions to the Ricci flow on certain threemanifolds, arXiv:math/0307245 (2003).

[RU98] A. Ros and F. Urbano, Lagrangian submanifolds of $\mathbf{C}^{n}$ with conformal Maslov form and the Whitney sphere, J. Math. Soc. Japan 50 (1998), no. 1, 203-226.

[SW01] R. Schoen and J. Wolfson, Minimizing area among Lagrangian surfaces: the mapping problem, J. Differential Geom. 58 (2001), no. 1, 1-86.

[SW03] - Mean curvature flow and Lagrangian embeddings, Preprint (2003).

[Smo96] K. Smoczyk, A canonical way to deform a Lagrangian submanifold, arXiv:dgga/9605005 (1996).

[Smo99] - Harnack inequality for the Lagrangian mean curvature flow, Calc. Var. Partial Differential Equations 8 (1999), no. 3, 247-258.

[Smo00] - The Lagrangian mean curvature flow (Der Lagrangesche mittlere Krümmungsfluß, Leipzig: Univ. Leipzig (Habil.), 102 S. (2000).

[Smo02] - Angle theorems for the Lagrangian mean curvature flow, Math. Z. 240 (2002), no. $4,849-883$.

[Smo04] L L L L L L existimence of the Lagrangian mean curvature flow, Calc. Var. Partial Differential Equations 20 (2004), no. 1, 25-46.

[Smo05] , Self-shrinkers of the mean curvature flow in arbitrary codimension, Int. Math. Res. Not. 48 (2005), 2983-3004.

[SW02] K. Smoczyk and M.-T. Wang, Mean curvature flows of Lagrangians submanifolds with convex potentials, J. Differential Geom. 62 (2002), no. 2, 243-257.

[SW11] Generalized Lagrangian mean curvature flows in symplectic manifolds, Asian J. Math. 15 (2011), no. 1, 129-140.

[Sta98] N. Stavrou, Selfsimilar solutions to the mean curvature flow, J. Reine Angew. Math. 499 (1998), 189-198.

[Sto94] A. Stone, A density function and the structure of singularities of the mean curvature flow, Calc. Var. Partial Differential Equations 2 (1994), no. 4, 443-480.

[Tem76] R. Temam, Applications de l'analyse convexe au calcul des variations, Nonlinear operators and the calculus of variations (Summer School, Univ. Libre Bruxelles, Brussels, 1975), Springer, Berlin, 1976, pp. 208-237. Lecture Notes in Math., Vol. 543 (French).

[TY02] R. P. Thomas and S.-T. Yau, Special Lagrangians, stable bundles and mean curvature flow, Comm. Anal. Geom. 10 (2002), no. 5, 1075-1113.

[TW04] M.-P. Tsui and M.-T. Wang, Mean curvature flows and isotopy of maps between spheres, Comm. Pure Appl. Math. 57 (2004), no. 8, 1110-1126. 
[Wan01a] M.-T. Wang, Mean curvature flow of surfaces in Einstein four-manifolds, J. Differential Geom. 57 (2001), no. 2, 301-338.

[Wan01b] — Deforming area preserving diffeomorphism of surfaces by mean curvature flow, Math. Res. Lett. 8 (2001), no. 5-6, 651-661.

[Wan02] Long time existence and convergence of graphic mean curvature flow in arbitrary codimension, Invent. Math. 148 (2002), no. 3, 525-543.

[Wan03] , Gauss maps of the mean curvature flow, Math. Res. Lett. 10 (2003), no. 2-3, 287-299.

[Wan04] The mean curvature flow smoothes Lipschitz submanifolds, Comm. Anal. Geom. 12 (2004), no. 3, 581-599.

[Wan05] - Subsets of Grassmannians preserved by mean curvature flows, Comm. Anal. Geom. 13 (2005), no. 5, 981-998.

[Wan08a] _ A convergence result of the Lagrangian mean curvature flow, Third International Congress of Chinese Mathematicians. Part 1, 2, AMS/IP Stud. Adv. Math., 42, pt. 1, vol. 2, Amer. Math. Soc., Providence, RI, 2008, pp. 291-295.

[Wan08b] Lectures on mean curvature flows in higher codimensions, Handbook of geometric analysis. No. 1, Adv. Lect. Math. (ALM), vol. 7, Int. Press, Somerville, MA, 2008, pp. 525-543.

[Whi05] B. White, A local regularity theorem for mean curvature flow, Ann. of Math. (2) 161 (2005), no. 3, 1487-1519.

[Whi09] Currents and flat chains associated to varifolds, with an application to mean curvature flow, Duke Math. J. 148 (2009), no. 1, 41-62.

[Xin08] Y. Xin, Mean curvature flow with convex Gauss image, Chin. Ann. Math. Ser. B 29 (2008), no. 2, 121-134.

Leibniz Universität Hannover, Institut für Differentialgeometrie, Welfengarten 1, 30167 Hannover, Germany

E-mail address: smoczyk@math.uni-hannover.de 